\title{
Building Up and Wearing Down Episodic Memory: Mnemonic Discrimination and Relational Binding
}

\author{
Chi T. Ngo \\ Temple University
}

\author{
Ying Lin \\ University of Rochester
}

\author{
Nora S. Newcombe and Ingrid R. Olson \\ Temple University
}

\begin{abstract}
Our capacity to form and retrieve episodic memories improves over childhood but declines in old age. Understanding these changes requires decomposing episodic memory into its components. Two such components are (a) mnemonic discrimination of similar people, objects, and contexts, and (b) relational binding of these elements. We designed novel memory tasks to assess these component processes using animations that are appropriate across the life span (ages 4-80 in our sample). In Experiment 1, we assessed mnemonic discrimination of objects as well as relational binding, in a common task format. Both components follow an inverted U-shaped curve across age but were positively correlated only in the aging group. In Experiment 2, we examined mnemonic discrimination of context and its effect on relational binding. Relational memory in low-similarity contexts showed robust gains between the ages of 4 and 6, whereas 6-year-olds performed similarly to adults. In contrast, relational memory in high-similarity contexts showed more protracted development, with 4- and 6-year-olds both performing worse than young adults and not differing from each other. Relational memory in both context conditions declined in aging. This multiprocess approach provides important theoretical insights into life span changes in episodic memory.
\end{abstract}

Keywords: episodic memory, life span, mnemonic discrimination, relational binding

Episodic memory binds together the people, objects, and locations that make up the specific events of our lives, forming multielement traces of experiences that can guide ongoing behavior, help imagine the future, and enhance well-being. For instance, a person may often take walks on the beach, but an episodic memory would entail remembering a particular episode, for ex-

This article was published Online First March 21, 2019.

Chi T. Ngo, Department of Psychology, Temple University; Ying Lin, Brain and Cognitive Sciences, University of Rochester; Nora S. Newcombe and Ingrid R. Olson, Department of Psychology, Temple University.

Chi T. Ngo and Ying Lin contributed equally to this study.

Special thanks to Jelani Medford, Elizabeth Eberts, Sydnee Jacqueline, Jacqueline Pizsker, Melanie Dippolito, Jessica Palmarini, Ai Leen Oon, and Catherine Meehan for their assistance with data collection, and Duy An Phan for the voice recording of the animations. This work was supported by the National Institutes of Health (F31HD090872 to Chi T. Ngo), the National Science Foundation (SBE-1041707 to Nora S. Newcombe), and the National Institution of Mental Health (RO1 MH091113 to Ingrid R. Olson).

Data from Experiments 1 and 2 have been made publically available through the Open Science Framework at https://osf.io/s36gu/ and https:// osf.io/bc4qg/.

Correspondence concerning this article should be addressed to Chi T. Ngo, Department of Psychology, Temple University, 1701 North 13th Street, Philadelphia, PA 19123. E-mail: chi.ngo@temple.edu ample, walking with her younger sister on a beach on her birthday and finding a distinctive seashell. Successful recollection of distinct experiences relies on mnemonic discrimination among elements of similar episodes, for example, younger versus older sister, or ocean beach versus bayside beach, or which kind of seashell. Another key characteristic of such an episodic memory is relational binding, the construction and retrieval of representations that link together multiple elements within an event, for example, sister and seashell, seashell and sister's birthday (Horner \& Doeller, 2017; Tulving, 2002).

Computational models of memory posit that the hippocampal learning system instantiates computations critical for episodic memory capacity by performing both mnemonic discrimination and relational binding, through distinct neurobiological mechanisms that rely on different hippocampal subfields (Norman \& O'Reilly, 2003). The dentate gyrus is well suited to perform extreme pattern separation by assigning distinct representations to similar inputs via sparse coding. Thus, this function supports mnemonic discrimination by decreasing the degree of representational similarity among overlapping experiences. On the other hand, recurrent connectivity in active CA3 units serves to bind elements of an event to a shared representation, thus accomplishing relational binding to support episodic memory. Therefore, hippocampal coding schemes of pattern separation and conjunctive coding serve to (a) retain distinctiveness of a specific past experience, and (b) binding together constituents of an episodic memory, respectively. A large literature from com- 
putational modeling (O'Reilly \& McClelland, 1994; Rolls, 1996), animal models (e.g., Leutgeb, Leutgeb, Moser, \& Moser, 2007; McHugh et al., 2007), and neuroimaging in humans (Bakker, Kirwan, Miller, \& Stark, 2008; Chadwick, Bonnici, \& Maguire, 2014; Lacy, Yassa, Stark, Muftuler, \& Stark, 2011) supports this conceptualization.

Intriguingly, episodic memory has long been shown to be relatively poor in children (e.g., reviewed in Nelson, 1995; Olson \& Newcombe, 2014) and in older adults (e.g., reviewed in Devitt \& Schacter, 2016; for a meta-analysis, see Old \& Naveh-Benjamin, 2008). Below, we briefly review the literature on the life span patterns of mnemonic discrimination and relational binding processes.

\section{Mnemonic Discrimination}

Accurate episodic memory requires remembering details with high specificity, such that they can be distinguished from other similar memories. Mnemonic discrimination involves reducing the extent of overlap between similar inputs so as to circumvent catastrophic interference (McClelland, McNaughton, \& O'Reilly, 1995; Norman \& O'Reilly, 2003). One paradigm designed to assess this component of episodic memory is the Mnemonic Similarity Task (MST), in which target objects must be discriminated from perceptually similar objects (e.g., Kirwan \& Stark, 2007). Success in this task seems to rely on the hippocampus, particularly the DG/CA3 subfields (e.g., Lacy et al., 2011; Reagh \& Yassa, 2014).

Although recognition memory for distinct individual items (e.g., dog vs. chair) appears to develop early on (reviewed in Olson \& Newcombe, 2014) and shows relatively less drastic decline in aging (Naveh-Benjamin, 2000), mnemonic discrimination between similar elements (sometimes referred to as pattern separation) varies substantially across the life span. This question has been most studied in the aging population. A fundamental feature of memory impairment in older adults is difficulty in discrimination of similar items, suggesting that age-related decrements in mnemonic discrimination may underlie poor episodic memory in the elderly (Dennis, Bowman, \& Peterson, 2014; Stark, Yassa, Lacy, \& Stark, 2013; Yeung, Ryan, Cowell, \& Barense, 2013). In childhood, mnemonic discrimination using a child-friendly version of the MST improved between the ages of four and six years ( $\mathrm{Ngo}$, Newcombe, \& Olson, 2018). Unlike 6-year-olds and young adults, 4-year-olds showed a strong tendency to overgeneralize such that they were more likely to misidentify lures as old items than correctly identifying them as similar. Although 6-year-olds did not show a tendency to overgeneralize, their lure discrimination performance did not exceed chance level, whereas young adults did. Recent findings supports the idea of mnemonic discrimination continues to improve after age 6, with age-related differences found between children aged 5-6 and 11-12, and between children aged 8-9 versus young adults (Rollins \& Cloude, 2018).

\section{Relational Binding}

Relational binding is typically tested using tasks that assess memory for the co-occurrence of multiple unrelated items. A large literature has shown that relational binding undergoes robust improvements in early childhood (Lloyd, Doydum, \& Newcombe, 2009; Ngo et al., 2018; Olson \& Newcombe, 2014; Riggins, 2014;
Sluzenski, Newcombe, \& Kovacs, 2006; Yim, Dennis, \& Sloutsky, 2013) and significantly deteriorates in aging (Devitt \& Schacter, 2016; Old \& Naveh-Benjamin, 2008). Poor performance on relational memory tasks by young children (Lloyd et al., 2009; Ngo et al., 2018; Sluzenski et al., 2006) and older adults (Associative Deficit Hypothesis: Naveh-Benjamin, 2000) results from a combination of lower accuracy and higher false memory. Specifically, both young children ( $~ 4$ years of age; Lloyd et al., 2009; Ngo et al., 2018; Sluzenski et al., 2006; Yim et al., 2013) and older adults (Castel \& Craik, 2003; reviewed in Devitt \& Schacter, 2016; for a meta-analysis, see Old \& Naveh-Benjamin, 2008) are more likely to misidentify novel combinations of items from different pairs as an old combination.

A limitation of prior work is that studies to date have used different tasks for different age groups. Nevertheless, these results lend some support to the notion that the age-related improvement and decline in relational binding may be a crucial aspect of the development and senescence of episodic memory over the life span.

\section{Relations Between the Two Components}

Both mnemonic discrimination and relational binding have been mechanistically linked to the hippocampus (Davachi, 2006; Norman \& O'Reilly, 2003), and both are critical for recalling the richness of interpersonal experiences throughout the life span.

The neural correlates of each component may follow distinct maturational profiles in childhood, expressed in uncorrelated development between the two processes in childhood. Previous work on nonhuman primates has shown that the dentate gyrus is the most late-developing subfield relative to other subfields such as CA1-4 (Lavenex \& Banta Lavenex, 2013; Serres, 2001). Corroborating these findings, studies in humans have also shown that the protracted development of the dentate gyrus and CA3 extend well into young adulthood (e.g., Keresztes et al., 2017). However, we know little about how fine-grained discrimination and relational binding relate to each other behaviorally. If the relevant computations are carried out by distinct neuroanatomical substrates in different hippocampal subfields, performance on the two components may not be related. In fact, in an initial study, and somewhat surprisingly, Ngo et al. (2018) found that they were uncorrelated in 4- and 6-year-old children, and in young adults. It is important to note, however, that in that study relational binding and mnemonic discrimination were assessed using two different tasks with potential uneven task-specific difficulties unrelated to the central constructs. In older adults, the only available evidence is that mnemonic discrimination on the MST performance positively correlated with performance on an standardized episodic memory task called the Rey Auditory Verbal Learning Test (RAVLT; Stark et al., 2013), which requires participants to remember the association of words with a specific list, that is, source memory. The positive correlation between the two tasks is consistent with the idea that cognitive abilities tend to be more highly correlated in aging populations compared with young adulthood (Lövdén, Ghisletta, \& Lindenberger, 2004). This sparse evidence suggests the intriguing possibility that mnemonic discrimination and relational binding are uncorrelated in child development and early adulthood, but that later declines dovetail.

However, studies of children and the elderly have proceeded in relative isolation from each other, in part because of the absence of 
tasks appropriate for a wide age range. Therefore, the extent to which the two components develop in childhood and deteriorate in aging in a parallel or joint manner is unknown. Leveraging the fact that both mnemonic discrimination and relational binding undergo robust changes in human ontogeny and aging, testing them simultaneously in a life span sample will help to elucidate how the core properties of episodic memory are linked or dissociated in development and in aging.

Furthermore, most researchers focus on only one of the two components of episodic memory, either mnemonic discrimination or relational binding, thus preventing insights into the relations between these two core processes, that is, into the cognitive structure of episodic memory. The present experiments aimed at correcting both problems by using engaging animation videos that allow for encoding and testing of relational binding and mnemonic discrimination within the same paradigm. Importantly, our tasks can be used in a life span sample, ages ranged from 4 to 80 .

\section{Current Research}

We examined two key components of episodic memory-mnemonic discrimination and relational binding-across development and aging. In two experiments, relational binding was tested using an $\mathrm{AB}-\mathrm{AC}$ relational structure; that is, item $\mathrm{A}$ was paired with $\mathrm{B}$ in one context, and item $\mathrm{A}$ was paired with $\mathrm{C}$ in another context. In Experiment 1, we indexed mnemonic discrimination at the item level by using perceptually similar object exemplars. In Experiment 2 , we turned our attention to mnemonic discrimination at the context level. Context discrimination has been much less studied than discrimination between items but is imperative given the pivotal role of spatial context in episodic memory (Robin, 2018).

\section{Experiment 1}

Experiment 1 had two main goals. First, we aimed to characterize age-related differences in item-level mnemonic discrimination and relational memory across early childhood and aging. Second, we asked whether performances on these two components relate to each other differentially across ages. We tested 4-yearolds, 6-year-olds, young adults (ages 18-34), and older adults (ages 65-80) in a novel task that allowed us to assess both mnemonic discrimination and relational binding, while equating the encoding phase and the test format to minimize methodological differences in measuring the two processes. The encoding phase was consisted of four animations, each providing a narrated tour of two different locations. Each location contained eight associations with an $\mathrm{AB}-\mathrm{AC}$ relational structure. Each association comprises one overlapping element (an item seen in both location [A]), and a unique element (an item seen in only one location $[\mathrm{B} / \mathrm{C}]$ ). The test phase consisted of four-alternative forced choice trials, equally divided between Item Mnemonic Discrimination and Relational Binding test trials. The only differences between the Item Mnemonic Discrimination and Relational Binding test trials were the types of lures presented along side the targets. One important aspect of this design is that it allowed us to examine both accuracy and error rates that reflect the integrity of each component. Whereas overall accuracy provides a gross index memory performance, the types of memory errors can elucidate the specific aspect of episodic memory. Crucially, this task is appropriate for a wide age range (ages 4-80), as we found no floor or ceiling effects. To our knowledge, there is no equivalent task in the existing memory literature.

\section{Method}

Participants. A total of 32 four-year-old (15 females; $M_{\text {month }}=53.49 \pm 3.54$ ) children and 32 six-year-old (13 females; $\left.M_{\text {month }}=76.01 \pm 3.55\right)$ children recruited from the Philadelphia suburbs participated in the study at the Temple Ambler Infant and Child Laboratory. Children recruited were free of color-blindness and psychological, neurological, and developmental disorders as reported by a parent. Informed consent was obtained from the child's parents. Two additional 4-year-old children participated but were not included in the data analyses because of incomplete procedure $(n=1)$ and experimenter error $(n=1)$.

The young adult sample consisted of 32 undergraduate students (21 females; $M_{\text {age }}=21.59 \pm 3.30$; range $=18-34, M_{\text {education }}=$ 12-16 years) from Temple University. Thirty-two older adults (20 females; $M_{\text {age }}=71.31 \pm 4.84$; range $=65-80$ ) who enrolled in Temple University's Osher Lifelong Learning Institute participated in the study. The average number of years of education for older adults was $18.14 \pm 3.87$, range $=12-28$. Older adults were also screened for cognitive impairment with the Mini Mental State Examination (MMSE, Folstein, Folstein, \& McHugh, 1975)—a brief test that is widely used to screen for dementia. The average score was $28.59 \pm 1.78$, the range was $24-30$, with 24 being set as the cutoff, to minimize to risk of including older adults with preclinical dementia (Tombaugh \& McIntyre, 1992). Young and older adults gave informed consent and reported having normal or corrected-to-normal vision.

All children were given a small toy for their participation; all young adults were given partial course credit; all older adults volunteered to participate in the study. This experiment was approved by the Temple University Institutional Review Board committee.

Overall procedure. The procedure was identical for children and young adults. In addition to the memory task, older adults were also administered three other tasks: the MMSE to screen for general cognitive status, the American National Adult Reading Test (AMNART; Grober \& Sliwinsk, 1991), which provided a measure of verbal intelligence, and the perceptual discrimination task. Older adults were always administered the MMSE and AMNART first, followed by the memory task, followed by the perceptual discrimination task.

\section{Memory task.}

Materials. The to-be-remember stimuli were animations created in Adobe Photoshop CS6 and Microsoft PowerPoint v14. These animations were designed to be engaging to all age ranges (examples of the animations can be viewed at https://sites.temple .edu/cnltu/people/zoe-ngo/). Four primary animation sequences were created: a house, park, ocean, and fair animations. Each animation consisted of a tour of two locations (e.g., a red and a blue house), which had unique background colors and distinct decorative details. Each location contained eight associations, totaling to 16 associations per animation. Every association was made up of one common item - an item that appeared in both locations, and a unique item-an item that only appeared in one location (see Figure 1). A total of 32 animations were created to 


\section{ENCODING}

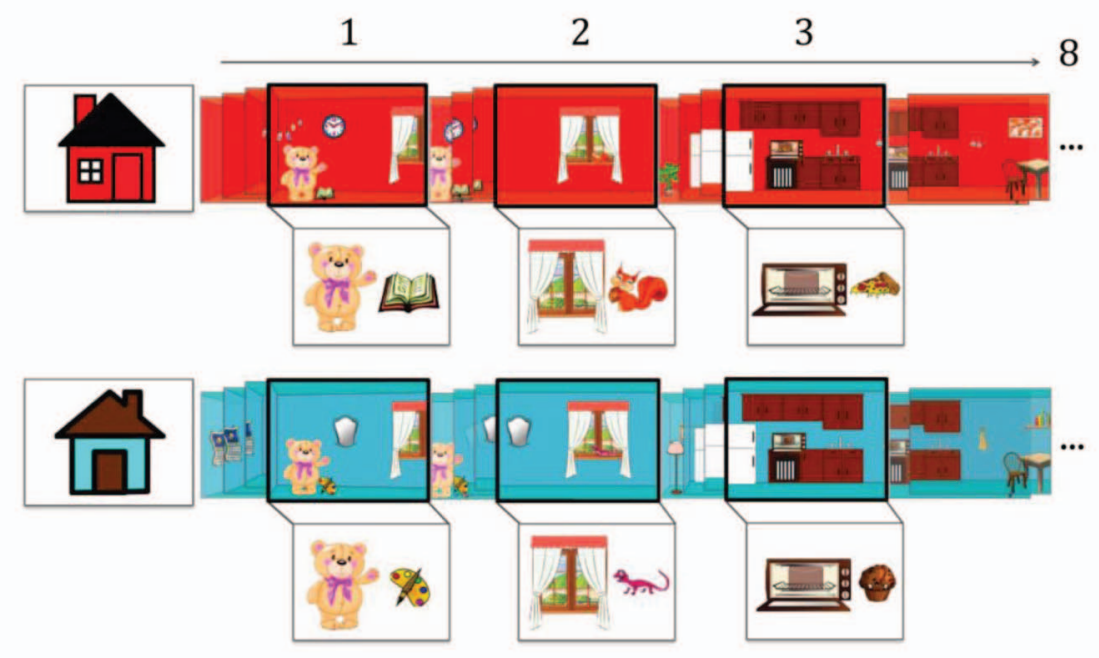

TEST
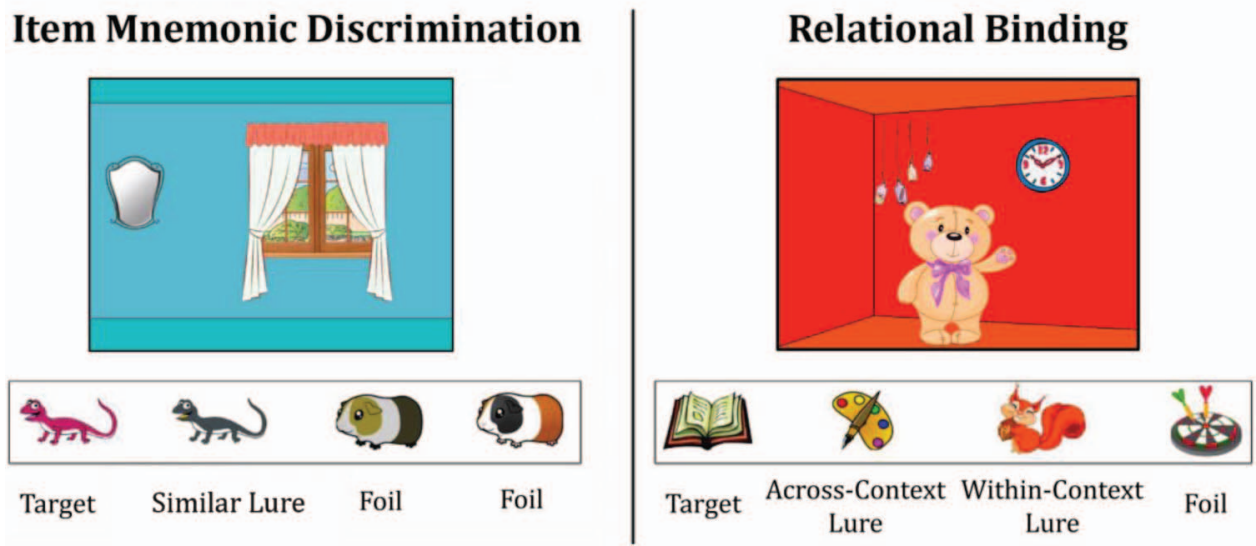

Figure 1. A schematic depiction of the associations presented in the encoding (top) and test (bottom) phases. At encoding, participants learned $16 \mathrm{AB}-\mathrm{AC}$ associations per animation. The test phase consisted of two types of test trials: item mnemonic discrimination and relational binding trials. See the online article for the color version of this figure.

counterbalance the unique items and the order in which the locations were visited.

Procedure. All participants were tested individually and were randomly assigned to different animation and test versions. For each animation, participants followed an encoding-test procedure.

Encoding. At the beginning of each animation, a female voice recording instructed participants that they would watch an animation that toured two different locations (e.g., two houses/parks) and that they would have to remember the things they see in these places. For instance, the house animation toured two different colored houses: a red house and a blue house. Eight associations (e.g., bear-book; window-lizard) were sequentially shown along the tour in each house. Each association comprised one common element (present in both locations; e.g., bear) and one unique element (present in only one location; e.g., book; see Figure 1, top). The appearances of the unique items were always accompanied by a chime sound, implemented to signal that a unique item appeared on the screen. Each association was presented statically for $5 \mathrm{~s}$ with 12 transition frames $(100 \mathrm{~ms} /$ frame $)$ before the next association appeared. The order of association (bear-[book/paint; window-[lizard/squirrel] was matched between the two contexts. A female voice narrated each tour (e.g., "First, we are going to the red house") and a child-friendly nonverbal music track played throughout the animation. In sum, participants viewed 16 associations in each animation, equally divided between the two locations visited in a given animation. Each animation was only seen once and lasted approximately $5 \mathrm{~min}$. The order of the two locations in each animation and the order of the four animations were counterbalanced across participants.

Test. The test phase immediately followed the encoding phase of each animation and consisted of 16 four-alternative-forcedchoice trials per animation. Among the 16 test items, eight were assigned as Item Mnemonic Discrimination test trials (Cronbach's alpha $=.89$ ) and the other eight as Relational Binding test trials 
(Cronbach's alpha $=.90)$. Among the eight associations in each location, four were randomly assigned as Item Mnemonic Discrimination test trials and the other four as Relational Binding test trials. The test trials for each animation were presented in a randomized order. Participants were randomly assigned to one of the two test versions created for each animation. The two test versions differed such that the test items assigned as the Item Mnemonic Discrimination trials in Version 1 were assigned as the Relational Binding trials in Version 2 and vice versa.

In both types of test trials, participants were presented with a static screenshot of the common item in a location (e.g., bear in the red house), matched with the screenshot of when the unique item first appeared in the animation, with four options shown beneath. In the Item Mnemonic Discrimination test trial, the four options included: a target, a similar lure, and two object exemplars of foils. Targets were the correct unique items, for example, the identical lizard paired with the window in the blue house. Similar lures were items that were perceptually similar to the target, but not identical to the target, for example, a similar lizard. Similar lures were always exemplars that differed on the color dimension from targets. Foils were novel items that never appeared in the animation. In addition to overall accuracy, this design also allowed us to operationalize two types of errors: (a) item memory errors occurred when the foil was selected, indicating that participants did not distinguish old from novel items; and (b) mnemonic discrimination errors occurred when the perceptual lure was selected, indicating that participants did not remember targets with high resolution and confused a perceptually similar exemplar to an old item.

In the Relational Binding test trials, the four options included: a target, an across-context lure, a within-context lure, and a foil. Targets (e.g., book) were the correct unique items paired with the common element (bear) in a specific location (red house). Acrosscontext lures (e.g., paint) were the unique items paired with the common element, but seen in the other location (blue house). Within-context lures (e.g., squirrel) were unique items seen in the correct location (red house), but were not paired with the common element. Foils were novel items that were not seen at encoding.

Similarly, we operationalized three types of errors: (a) item memory errors occurred when the foil was selected, resulting from the fact that participants did not distinguish old from new items, (b) interitem binding errors occurred when within-context lures were selected, indicating that participants did not correctly bind the common item to its paired unique item; and (c) association-context binding errors occurred when across-context lures were selected, indicating that participants did not bind the common-unique pairs to its context.

Participants were asked to choose the item that they saw with a given scene by pointing to one of the four options presented on the screen (see Figure 1, bottom). The experimenter recorded participants' responses on paper. For both test trial types, the locations of the four test items were counterbalanced across test trials. All unique items were counterbalanced such that they were assigned as each test item type an equal number of times across participants. The task lasted approximately $30 \mathrm{~min}$.

Perceptual discrimination task. Immediately after the memory task, older adults were tested on their abilities to perceptually discriminate similar lures to rule out the possibility that age-related decline in visual acuity could contaminate our results. The task consisted of 32 pairs of perceptually similar exemplars interspersed with 16 catch trials (pairs of identical items). Each of the 32 pairs of similar exemplars contained a target and a corresponding similar lure selected from the item mnemonic discrimination test trials presented next to each other on a desktop screen. Older adults were instructed to provide a yes/no verbal response to whether the two items on the screen were identical to each other. All older adults performed at $100 \%$ accuracy on this task, ruling out the possibility that any age-related differences in perceptual discrimination may account for the age effect in mnemonic discrimination accuracy.

Verbal intelligence: American National Adult Reading Test (AMNART; Grober \& Sliwinsk, 1991). Because our older adult sample was recruited from the community and thus less homogeneous than our young adult sample who were all undergraduate students, verbal intelligence was estimated in older adults using the 45-item AMNART (an American version of the National Adult Reading Test [Nelson, 1995]). This test measures the ability to read aloud irregular words. Pronunciation errors were tallied and AMNART-estimated IQ score was calculated using Grober and Sliwinsk's formula, which accounts for years of education ( $M$ score $=123.90, S E=0.96$, range $=107.19-130.64$ ).

\section{Results}

Overall accuracy did not differ among any of the animation sequences, or between the first and the last animations, all $p s>$ .05 , suggesting that there are no unintended differences in difficulty among the four animations, and that participants did not improve with practice or get worse from fatigue between the first and the last animations. No significant sex differences were found either in Item Mnemonic Discrimination or Relational Binding in any of the age groups (all $p s>.30$ ) and thus we collapsed across sex in subsequent analyses.

Item mnemonic discrimination. The proportion of each test item selected across all Item Mnemonic Discrimination test trials was calculated for each participant. One-way ANOVAs were conducted to test age effects for each test item: target, similar lure, and foil (see Figure 2).

Accuracy. Accuracy-the proportion of trials in which the targets were selected-was affected by age, $F(3,124)=22.66$, $p<.001, \eta^{2}=0.35$. Tukey post hoc tests showed that 4 -year-olds chose targets less frequently than 6-year-olds, young adults, and older adults $(M \mathrm{~s}=.62 ; .86, .91$, and .85 ; all $p \mathrm{~s}<.001)$. Six-yearolds and young and older adults performed similarly, all $p s>.34$ Despite the fact that 4-year-olds performed worse than their older counterparts, they were able to remember the specific item that they encountered, even in the presence of a highly similar item, that is, their performance was significantly greater than chance, $t(31)=7.81, p<.001$.

Error rates. One-way ANOVAs also revealed significant age differences in mnemonic discrimination errors, $F(3,124)=13.75$, $p<.001, \eta^{2}=0.25$. Four-year-olds chose similar lures more often than all other age groups $(M \mathrm{~s}=.18 ; .08 ; .06 ; .11$ all $p \mathrm{~s}<.01)$. In contrast, the other three age groups performed comparably, all $p \mathrm{~s}>$.10. Similarly, the proportion of foils chosen also differed across age groups, $F(3,124)=20.50, p<.001, \eta^{2}=0.33$. Once again, 4-year-olds chose foils more often than all other age groups 
Item Mnemonic Discrimination

\section{Relational Binding}
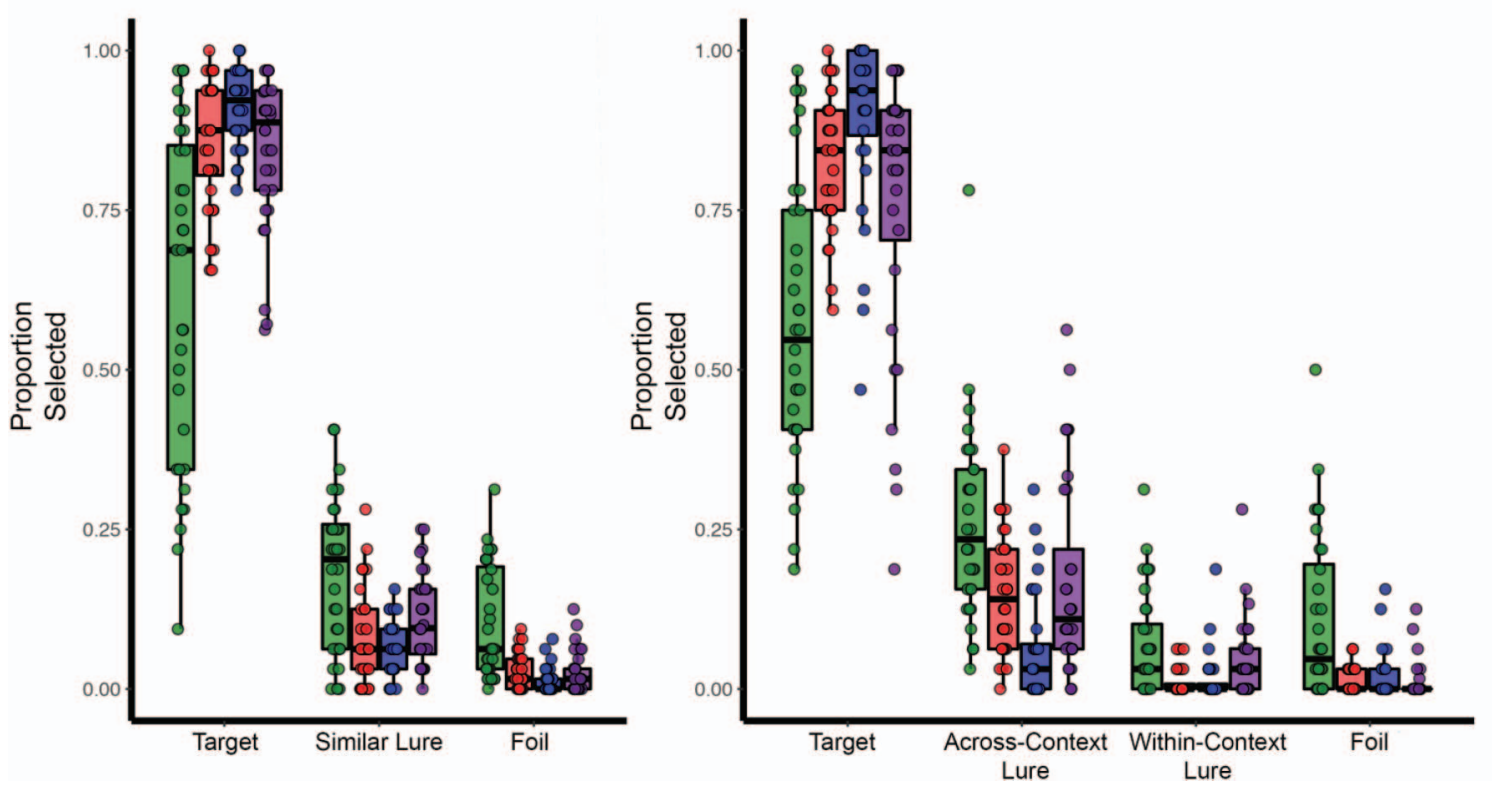

Figure 2. The distribution of proportion selected for each test items on the Item Mnemonic Discrimination test trials (left) and Relational Binding test trials (right) in four age groups. See the online article for the color version of this figure.

$(M \mathrm{~s}=.10 ; .03 ; .01 ; .04 ;$ all $p \mathrm{~s}<.001)$. In contrast, the other three age groups did not differ, all $p$ s $>.60$.

Overselection of foils indicates a potential problem with item memory, and thus in our next analysis we tested the effects of age on mnemonic discrimination accuracy, controlling for age differences in foil selection. The age effect in mnemonic discrimination accuracy remained significant, $F(3,123)=2.84, p=.04, \eta^{2}=$ .07 . Thus, the age effect in mnemonic discrimination cannot be accounted for by age differences in item memory.

Did participants make more mnemonic discrimination errors than item memory errors? Paired-sample $t$ tests showed this to be true: all age groups chose more similar lures than foils, all $p \mathrm{~s}<$ .001 .

Relational binding. The proportion of each test item selected across all Relational Binding test trials was calculated for each participant. One-way ANOVA was conducted to test age effect on each type of test items: target, across-context lure, within-context lure, and foil (see Figure 2).

Accuracy. Accuracy-the proportion of trials in which the targets were selected-was affected by age, $F(3,124)=20.89$, $p<.001, \eta^{2}=0.34$. The biggest and most consistent age difference was in 4-year olds. Tukey post hoc tests showed that 4-yearolds chose targets less frequently than 6 -year-olds, young adults, and older adults $(M \mathrm{~s}=.57 ; .83$; and $.90 ; .77$; all $p \mathrm{~s}<.001$. The addition of just two years of age dissolved this age difference: 6-year-olds performed no differently than young adults or older adults (all $p \mathrm{~s}>.36$ ). However, older adults performed worse than young adults, $p=.02$. It is worth noting that despite being the worst performing age group, 4-year-olds chose targets at a level significantly greater than chance performance of $0.25, t(31)=$ 8.16, $p<.001$. This demonstrates that even the young children were able to bind multiple items together and to a specific context, albeit showing lowest accuracy among four age groups.

Error rates. Across-context errors differed across age groups, $F(3,124)=12.93, p<.001, \eta^{2}=0.24$. Similar to the findings in the accuracy data, 4-year-olds chose across-context lure more often than 6-year-olds, young adults, and older adults $(M \mathrm{~s}=0.26$; $0.15 ; 0.07 ; 0.17$; all $p$ s $<.02$ ). Six-year-old performed comparably to older adults, $p=.94$, but made more across-context error than did young adults, $p=.04$. Relative to young adults, older adults also made more across-context errors, $p=.006$.

Within-context errors also differed across age groups, $F(2$, $93)=7.83, p<.001, \eta^{2}=0.16$. Four-year-olds made more within-context errors than 6-year-olds and young adults $(M \mathrm{~s}=$ $0.07 ; 0.01 ; 0.02$; all $p s<.001)$ but did not differ from older adults $(M=.05 ; p=.33)$. No differences were found among the other three age groups, all $p \mathrm{~s}>.06$.

The proportion of foils selected also differed as a function of age, $F(3,124)=14.50, p<.001, \eta^{2}=0.26$. Again, the poor performance of four year olds stood out. Four-year-olds chose foils more frequently than 6-year-olds, young adults, and older adults $(M \mathrm{~s}=.11 ; .01 ; .02 ; .02 ; p<.001)$ whereas the other three age groups did not differ (all $p \mathrm{~s}>.98$ ).

Similar to the Item Mnemonic Similarity test portion, we examined whether the age differences in relational binding accuracy were accounted for by age differences in item memory. We conducted a one-way ANCOVA on relational binding accuracy with the proportion of foils selected as a covariate. The effect of age on relational binding accuracy remained significant, $F(3,123)=$ 9.93, $p<.001, \eta^{2}=.20$, suggesting that the age effect in relational binding accuracy cannot be completely accounted for by the age differences in item memory. 
Next, we tested whether memory errors for association-context relational binding were higher than the other two types of errors by conducting one-way ANOVAs comparing the proportion of across-context lure, within-context lure, and foil selected by each age group. There was a significant main effect of error type in all age groups, all $p \mathrm{~s}<.001$. Across all ages, across-context error was higher than within-context error, all $p s<.001$, and item memory error, all $p s<.002$. The differences between within-context lures and foils were mixed. The two types of errors did not differ in either 6-year-olds or young adults, all $p \mathrm{~s}>.81$. However, 4-yearolds chose foils more often than within-context error, $p=.04$, whereas the opposite was found for older adults, $p=.002$.

In sum, these results suggest that the ability to bind associations to their contexts significantly improves between the ages of four and six, with more subtle changes evolving between the age of six and the beginning of adulthood. Peak performance is found at this age: older adults had relatively lower accuracy and made more across-context errors, reflecting deficits in contextual binding.

Individual differences in aging. The results of our prior analyses show that older adults performed similarly to 6-year olds. However, it is known that individual differences are magnified in older adults and that average cognitive performance differs between, for instance, 70-year-olds and 80-year-olds (Holden, Toner, Pirogovsky, Kirwan, \& Gilbert, 2013). Here we asked whether mnemonic discrimination and relational binding were affected by age in our older adult sample, using age as a continuous variable in a partial Pearson correlation. To ensure that general cognitive status and IQ did not contribute to the variance, MMSE and AMNART performance were used as covariates. As expected, age negatively correlated with performances on both tasks, $r(28)=-.57, p=.001$ and $r(28)=-.65, p<.001$, for mnemonic discrimination and relational binding accuracy, respectively (see Figure 3).

Item mnemonic discrimination and relational binding correlations. To examine whether item mnemonic discrimination and relational binding are related mnemonic processes, we tested whether the types of error indexing item mnemonic discrimination failure and relational binding failure, that is, across-context error, would relate to each other in each age group separately. Pearson correlations yielded nonsignificant correlations between mnemonic discrimination error and across-context error in the 4-year-olds, $r(30)=.03, p=.87,6$-year-olds, $r(30)=.02, p=$ .92 , and young adults, $r(30)=.17, p=.36$. In contrast, these two types of error showed a strong positive correlation in older adults, $r(30)=.66, p=<.001$, suggesting that older adults with greater item mnemonic discrimination deficit also had greater relational binding deficit (see Figure 4). To further examine whether the correlation between item mnemonic discrimination and relational binding in older adults is driven by age, cognitive status, and verbal IQ, we conducted a partial correlation with three control variables: age, MMSE, and AMNART. The partial correlation between across-context error and mnemonic discrimination error remained significant, $r(27)=.52, p=.004$.

Importantly, the correlation in older adults was significantly greater than that in 4-year-olds, $z=-2.90, p=.004,6$-year-olds, $z=-2.94, p=.003$, and young adults, $z=-2.37, p=.02$, suggesting a significant increase in dedifferentiation between the two mnemonic components in older adults.

\section{Discussion}

Experiment 1 employed the same engaging task for all participants to characterize the life span profile of two key components of episodic memory. Two kinds of findings were revealed: developmental curves for performance and relations between the components at various points in the life span.

Item mnemonic discrimination and relational binding performance. Between the ages of four and six, substantial changes occurred in the way children distinguish between similar memory traces. Our youngest children's memory appeared to lack granularity; that is, they were less able to correctly retrieve the identical item encountered in the presence of a similar lure. Mne-

\section{Item Mnemonic Discrimination}

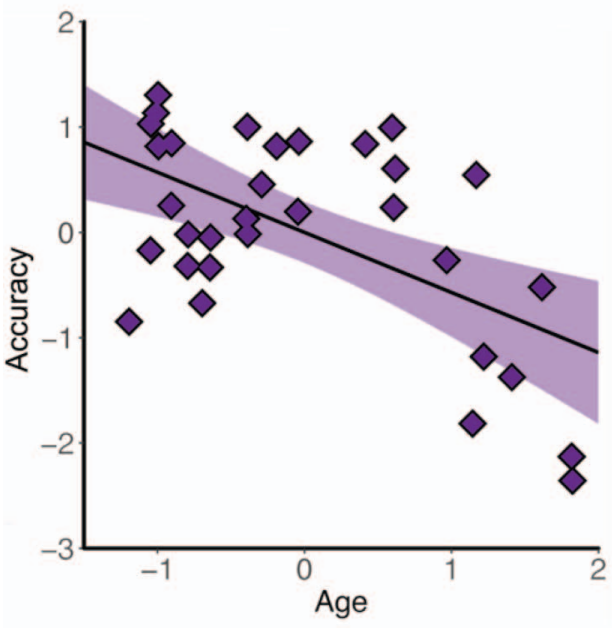

Relational Memory

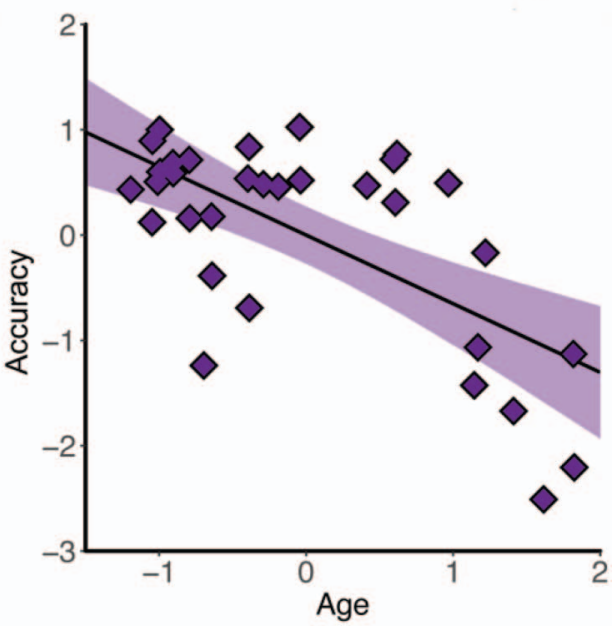

Figure 3. Scatterplots of the residuals illustrating the relation between age and item mnemonic discrimination accuracy (left) and between age and relational memory accuracy (right) in older adults, after partialing out MMSE and AMNART scores. See the online article for the color version of this figure. 

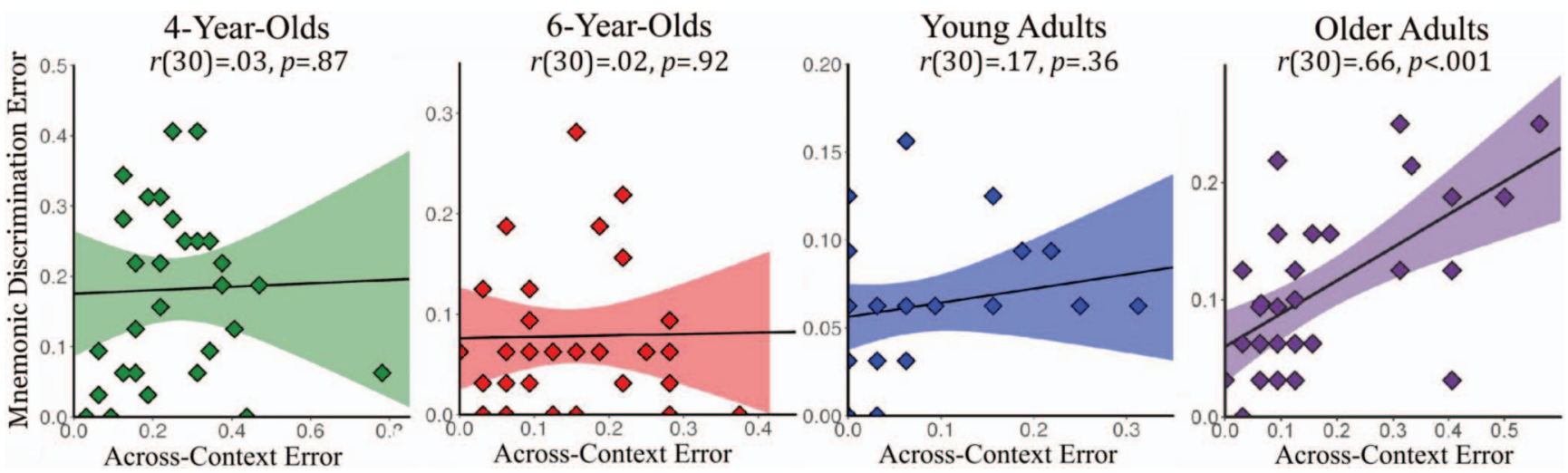

Figure 4. Scatterplots of across-context error ( $x$ axes) and mnemonic discrimination error $(y$ axes) in the four age groups. See the online article for the color version of this figure.

monic discrimination improved, and errors decreased, between the ages of four and six, whereas no differences were found among 6-year-olds, young adults, and older adults. The age patterns in children and young adults are consistent with our previous results on lure discrimination on the MST (Ngo et al., 2018). It is worth noting that although no age effect was detected at a group level between young and older adults, age was inversely correlated with item mnemonic discrimination within our older adult sample, consistent with the idea of heterogeneity among older adults (Holden et al., 2013; Stark et al., 2013).

Children's abilities to remember complex associations also appear to change substantially from the ages of 4 to 6 years. Our results show that relational binding performance, gauged in several different ways (e.g., accuracy, error rate) significantly improved during this age range. At the other end of the human life span, relational binding declined such that older adults had similar levels of relational memory as 6-year-olds, but worse than young adults. This inverted U-shape function has been reported previously for other domains of memory (item vs. item-location binding: Cowan, Naveh-Benjamin, Kilb, \& Saults, 2006; word pairs binding and monitoring: Fandakova, Shing, \& Lindenberger, 2013; reviewed in Shing et al., 2010). Among the older adults sample, more advanced age correlated with worse relational binding.

Correlations between item mnemonic discrimination and relational binding. How do item mnemonic discrimination and relational binding relate to one another across the life span? In three of the age groups tested (all children and young adults), mnemonic discrimination and relational binding were uncorrelated. These results conceptually replicate the results of our previous study, in which the two constructs were assessed using distinct tasks (Ngo et al., 2018). Our results also converge with a recent study showing behavioral dissociation between mnemonic discrimination on the MST and performance on a face-name relational binding task in children aged 6-14 (Keresztes et al., 2017). In this study, the authors also found that mnemonic discrimination was specifically related to the multivariate patterns of gray matter volumes across the hippocampal subfields, whereas relational memory indexed by a source judgment task was related to maturation of the frontal cortex gray matter volumes.

In contrast to these results, older adults' item mnemonic discrimination and relational binding were strongly correlated. Age, cognitive status, and verbal intelligence did not account for this correlation. Corroborating these results are prior studies showing that in older adults, MST lure discrimination positively correlated with performance on an episodic memory task called the Ray Auditory Verbal Learning Test (Stark et al., 2013). Accuracy on this task, similar to our relational binding task, requires intact item-source binding. Taken together, these findings indicate that item mnemonic discrimination and association-context relational binding may develop and remain independent from early childhood to adulthood; however, their senescence shares the same path.

In Experiment 1, we tested mnemonic discrimination for individual object stimuli, similar to most previous studies in humans (reviewed in Yassa et al., 2011). However, episodic memory is not only about remembering individual items; rather, the essence of an episodic memory is the capacity to encode, store, and retrieve the focal item or event in conjunction with the surrounding context. The question of how mnemonic discrimination for interitem associations occurring in similar contexts changes across the life span had yet to be examined, and thus was the focus of Experiment 2 .

\section{Experiment 2}

Given that the fundamental characteristic of episodic memory is its rich contextual representation, the extent to which interference stemmed from context similarity can muddle a memory for a specific past event is a pivotal topic for investigation. With a few exceptions, most prior studies have employed tasks that demand mnemonic discrimination for singular items such as individual objects or scenes (reviewed in Yassa et al., 2011). Several fMRI studies have employed paradigms with complex stimuli such as foreground events and spatial background (Chadwick et al., 2014), city layouts using virtual environments (e.g., Kyle, Stokes, Lieberman, Hassan, \& Ekstrom, 2015; Stokes, Kyle, \& Ekstrom, 2015), or sequences of scenes (Berron et al., 2016). These studies offer evidence to suggest that the hippocampal neural representations carry event- or environment-specific information. However, these investigations have been only been conducted in young adults. In contrast to the human literature, the animal literature has largely examined mnemonic discrimination by testing behaviors dependent on contextual discrimination, with context similarity varied 
parametrically (e.g., Leutgeb et al., 2007) or categorically (e.g., McHugh et al., 2007). To our knowledge, mnemonic discrimination for contextual information has not been tested in children or older human adults.

The goal of this experiment was to understand how context similarity impacted relational binding, and how these effects may vary as a function of age across the life span. Importantly, our empirical approach helped us make closer contact with empirical paradigms of pattern separation in studies using rodents, which require the animals to elicit distinct behaviors contingent on discrimination of similar contexts (McHugh et al., 2007; reviewed in França, Bitencourt, Maximilla, Barros, \& Monserrat, 2017; Hunsaker \& Kesner, 2013). Similar to Experiment 1, we created a memory paradigm in which participants learned a series of $\mathrm{AB}-\mathrm{AC}$ associations in two contexts. The critical manipulation was whether associations $\mathrm{AB}$ and $\mathrm{AC}$ were learned in two similar (e.g., two houses) or dissimilar contexts (e.g., a house and a park). Participants' abilities to correctly identify targets were contingent upon successful contextual discrimination between the two locations in which associations $\mathrm{AB}$ and $\mathrm{AC}$ were encountered. We reasoned that the demand for mnemonic discrimination would be greater for associations learned in similar contexts compared with those learned in dissimilar contexts. In addition, we asked how relational binding dependent on context discrimination improve in early childhood and decline with old age.

We predicted that the greater demand on mnemonic discrimination would impede relational binding overall, and that the developmental course of relational binding may differ as a function of context discrimination demand. Although we have consistently seen robust gains in relational binding between ages 4 and 6 , it is possible that relational binding combined with the need to resolve interference from similar contexts may develop later in childhood, and decline more drastically in aging. High-resolution memory for complex association-context representations may depend on a mature hippocampal circuitry and its connectivity with a network of cortical regions including the prefrontal and posterior parietal cortices. It is likely that the ability to separate complex relational structures learned in highly interfering contexts recruits top-down control processes such as retrieval monitoring and strategy, supported by the prefrontal cortex (Wheeler, Stuss, \& Tulving, 1997), as well as reinstating episodic vividness, supported by the posterior parietal cortex (Rugg \& King, 2018). White matter pathways linking the hippocampus and other MTL regions to cortical regions have prolonged maturational course and steep declines in aging (Lebel et al., 2012).

\section{Method}

Participants. Thirty-six 4-year-olds (15 females; $M_{\text {month }}=$ $52.77 \pm 6.23$ ), 33 six-year olds (18 females; $M_{\text {month }}=81.42 \pm$ 4.97), 32 young adults ( 24 females; $M_{\text {age }}=20.41 \pm 2.41$; range $=$ $18-29, M_{\text {education }}=12-18$ years), and 32 older adults ( 23 females; $M_{\text {age }}=73.06 \pm 5.52 ;$ range $=65-85, M_{\text {education }}=12-26$ years $)$ were recruited and compensated in the same manner as Experiment 1. Older adults were screened for cognitive impairments with the MMSE $(M=28.72 \pm 1.23$; range $=25-30)$. All participants gave informed consent and reported having normal or correct-tonormal vision. This experiment was approved by the Temple University Institutional Review Board committee.
Overall procedure. All participants were completed the verbal IQ test prior to the memory task. Older adults were additionally tested on the MMSE, which was always administered first.

Memory task.

Materials. The to-be-remembered stimuli were animations created in Adobe Photoshop CS6 and Microsoft PowerPoint v14. Eight primary animation sequences were created: houses 1 and 2, parks 1 and 2, shopping malls 1 and 2, and farmers' markets 1 and 2. Versions 1 and 2 for each of the location were made distinctive to one another in terms of their layouts with unique background features and decorative details. To create different context conditions, two primary animation sequences were connected to make one animation that consists of a tour to two different locations. In the similar context condition, two versions of the same location type would be connected (e.g., house 1-house 2). For the dissimilar context condition, two different locations were connected (e.g., house 1-park 2; shopping mall 1-farmers' market 2), such that the tour always consisted of a tour to one indoor and one outdoor location to maximize the dissimilarity between to two encoding contexts (see Figure 5). Each animation lasted $5 \mathrm{~min}$, and a total of 64 animations were created to counterbalance the unique items and the order in which the locations were visited. Examples of the animations can be viewed at https://sites.temple.edu/cnltu/people/ zoe-ngo/.

Procedure. All participants completed both the similar and dissimilar context conditions (order counterbalanced), and were randomly assigned to one of the 32 animation versions in each condition. Participants followed an encoding-test procedure for each context condition. At encoding, a female child voice recording instructed the participants that they would go on two different trips, and that they would have to remember the things they saw. Every animation toured two different locations, with eight associations in each location. Each association was made up of one common element and one unique element, that is, $\mathrm{AB}-\mathrm{AC}$ format (see Figure 5 top). Similar to Experiment 1, the order of association (girl-[glasses/wallet]; table-[tissue box/cake] was matched between the two contexts. In addition, a 2-min transition clip (e.g., walking on a street pavement) separated the two locations in each animation to clearly indicate that the two places were distinct.

The test phase immediately followed the encoding phase of each animation and was similar to the Relational Binding task portion in Experiment 1. Each test phase consisted of 16 four-alternativeforced-choice trials (similar context condition: Cronbach's alpha $=.77$; dissimilar context condition: Cronbach's alpha $=.90$ ) In the test trial, participants were presented with a static screenshot of the common item in a location, with four options shown beneath including a target, an across-context lure, a within-context lure, and a foil-defined in the same manner as Experiment 1 (see Figure 5, bottom). The test trials for each animation were presented in a randomized order. The positions of the four test items were counterbalanced and were assigned as each test item type an equal number of times across participants. This procedure was identical for all age groups except the young and older adults who were administered the AMNART instead of the KBIT. Older adults were also asked to complete the MMSE. The entire procedure lasted approximately 35 min for all age groups.

Verbal intelligence. All children were administered the Kaufman's Brief Intelligence Test, second edition (KBIT-2: Kaufman \& Kaufman, 1990), to assess general verbal intelligence. Children 


\section{ENCODING}

SIMILAR CONTEXT CONDITION

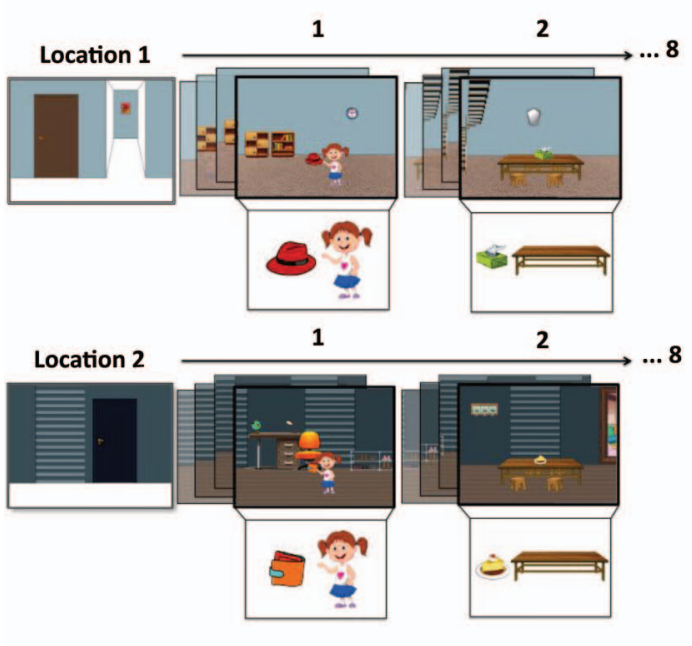

TEST

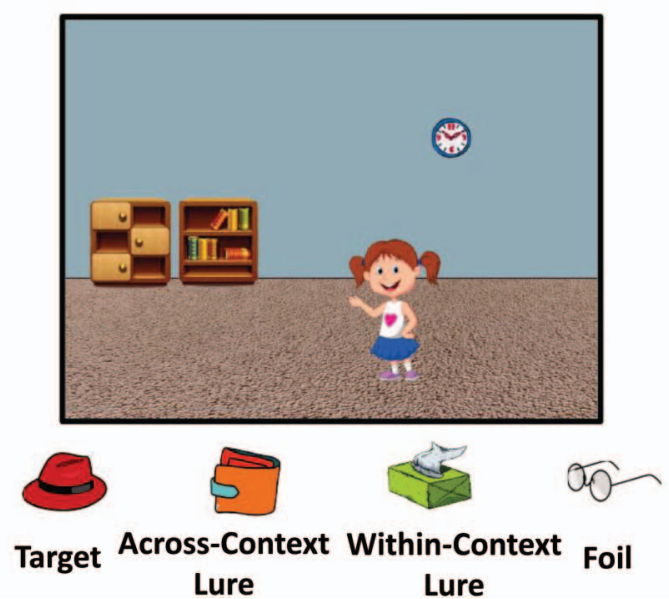

DISSIMILAR CONTEXT CONDITION
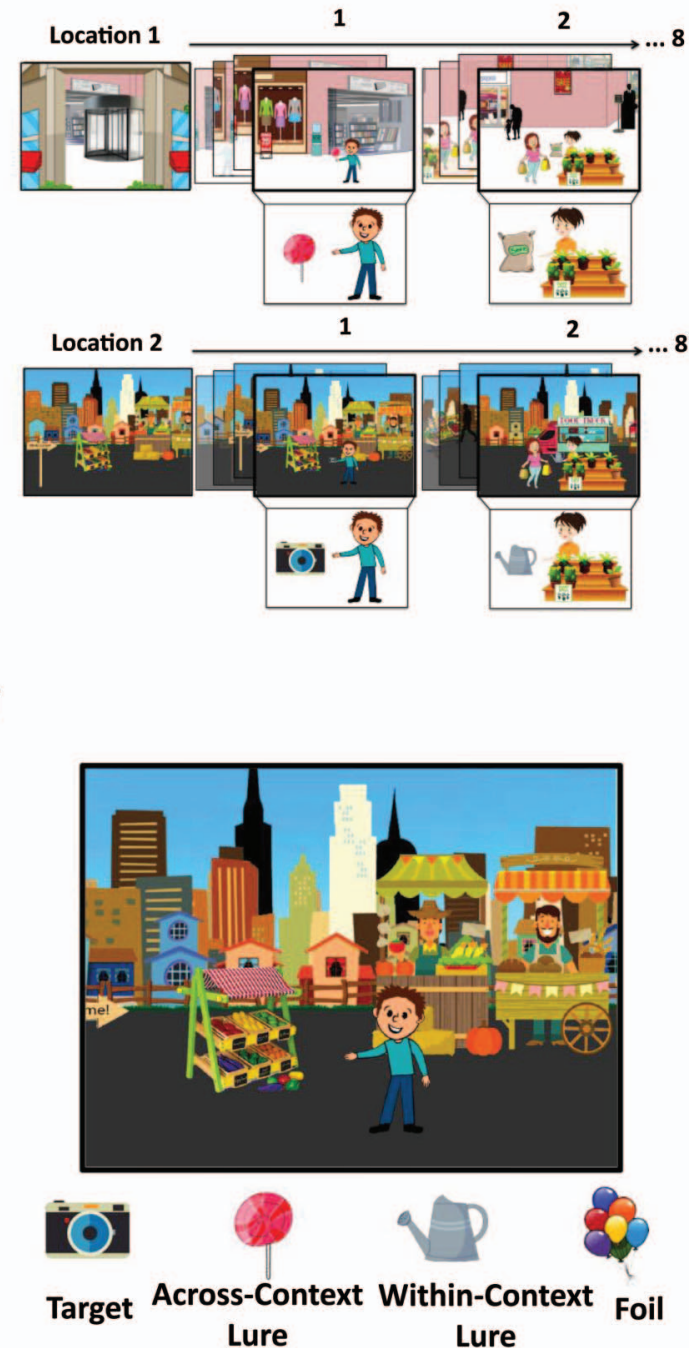

Figure 5. A schematic depiction of the associations presented in the encoding (top) and test (bottom) phases for the similar and dissimilar context conditions. See the online article for the color version of this figure.

were instructed to choose one of the six images simultaneously shown on a page that was the best match for a word or phrase (e.g., what has a skinny tails and squeaks? - a mouse), and to respond with a one-word answer to verbal riddles (e.g., what can only be seen at night and twinkles in the sky? — star, moon). The test, with increasing level of difficulty in each section, was terminated when a child provided four incorrect responses consecutively. Standard score was calculated for each child based on his or her age.

All young and older adults were administered the AMNART, described in Experiment 1.

\section{Results}

As in Experiment 1, performance was similar on the first and the second animations, $p=.71$. The order of context conditions to which participants were randomly assigned also did not affect accuracy, all $p$ s $>$.22. Last, across all age groups, males and females performed similarly, all $p \mathrm{~s}>.14$, thus we collapsed across sex in subsequent analyses. The proportion of test item selection for each context condition across four age groups are presented in Figure 6.

Relational memory performance. A 4 (age) $\times 2$ (context) repeated ANOVA was conducted separately for target, acrosscontext lure, within-context lure, and foil.

Accuracy. There was a main effect of age, $F(3,131)=28.44$, $p<.001, \eta^{2}=0.39$, a main effect of context, $F(1,131)=42.60$, $p<.001, \eta^{2}=0.25$, and a significant Age $\times$ Context interaction, $F(3,131)=2.75, p=.045, \eta^{2}=0.06$, on accuracy. In the dissimilar context condition, 4-year-olds were less accurate than 6-year-olds, young adults, and older adults $(M \mathrm{~s}=0.56 ; 0.84 ; 0.95$; 0.81 ; all $p$ s $<.001)$. In contrast, 6-year-olds were as accurate as 


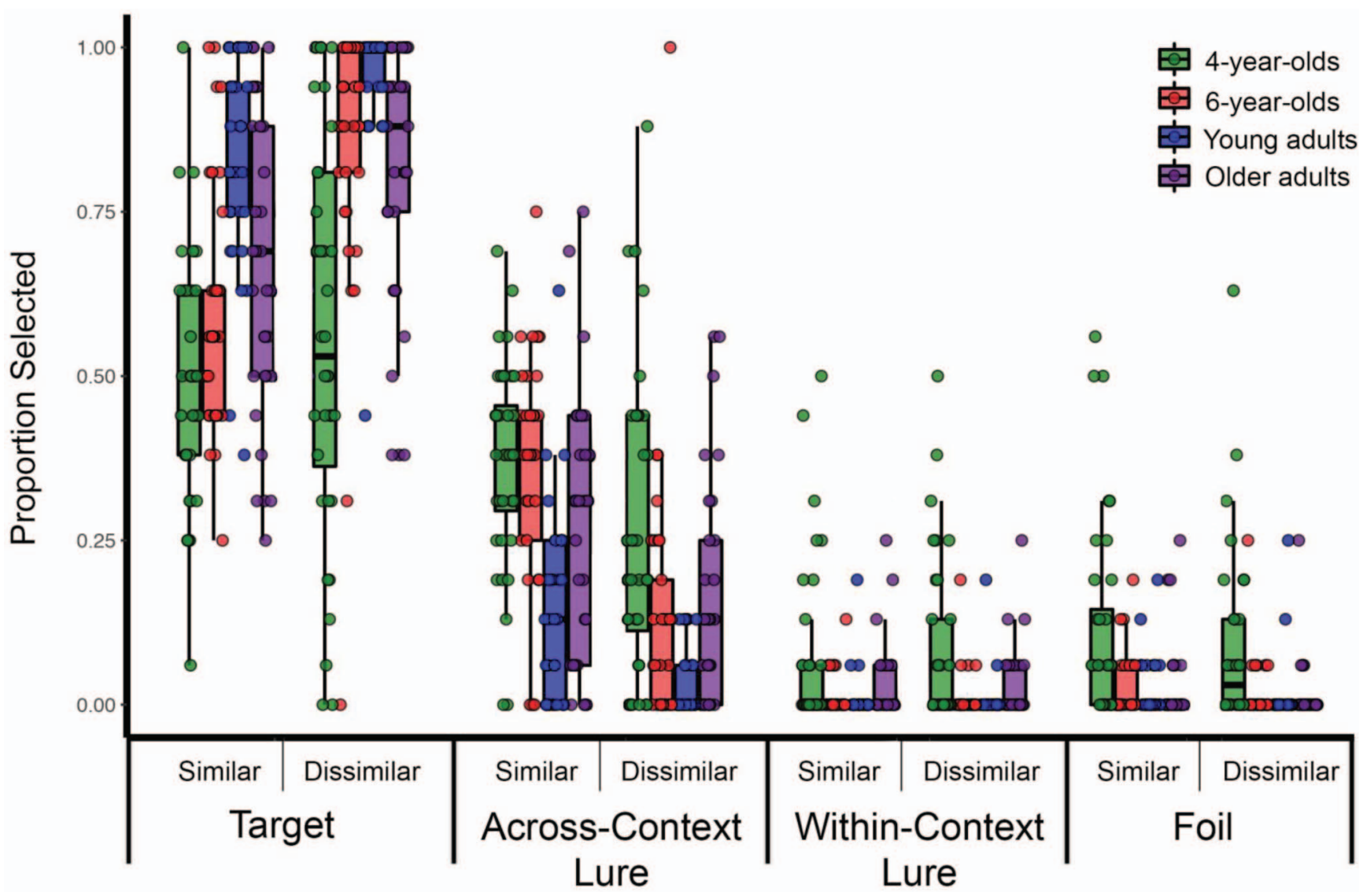

Figure 6. The distribution of proportion selected for each test item selected in the similar and dissimilar context conditions across four age groups. See the online article for the color version of this figure.

young and older adults, all $p \mathrm{~s}>.20$. Older adults performed worse than young adults, $p=.001$. These results are strikingly similar to the relational memory performance in Experiment 1 . In contrast, in the similar context condition, 4-year-olds and 6-year-olds did not differ from each other, although we note a trend $(M \mathrm{~s}=0.50 ; 0.60$, $p=.09)$, and children from both age groups performed worse than young adults $(M=.82$, all $p$ s $<.001)$. Older adults $(M=0.67)$ performed worse than young adults, $p=.006$, comparably to 6 -year-olds, $p=.55$, and better than 4-year-olds, $p=.002$. The Age $\times$ Context interaction seemed to be primarily driven by the fact that the 6-year-olds performed similarly to young adults in the dissimilar context condition, but performed much worse than the young adults, and comparable with the 4-year-olds in the similar context conditions.

Four-year-olds, despite being the worst performing age group, chose targets at a level significantly greater than chance performance of 0.25 in both the dissimilar, $t(35)=6.14$, and similar, $t(35)=7.94$, context conditions, all $p \mathrm{~s}<.001$. Thus 4-year olds were able to bind multiple items together and to a specific context, even when the contexts are similar to each other.

Error rates. Across-context errors were affected by age, $F(3$, $131)=17.39, p<.001, \eta^{2}=0.29$, and by context condition, $F(1$, $131)=55.28, p<.001, \eta^{2}=0.30$, and the Age $\times$ Context interaction was not significant, although there was a trend, $F(3$,
131) $=2.15, p=.10, \eta^{2}=0.05$. Overall, 4-year-olds did not differ from 6-year-olds, $p=.14$, but performed worse than young and older adults, all $p \mathrm{~s}<.02$. Six-year-olds performed worse than young adults, $p<.001$, but performed comparably to older adults, $p=.80$. Older adults made more errors than young adults, $p=$ .001. As predicted, collapsed across age groups, across-context errors were lower in the dissimilar than the similar context condition.

Within-context errors were also impacted by age, $F(3,131)=$ $10.47, p<.001, \eta^{2}=0.19$. However, as expected, there was no main effect of context condition, $F(1,131)=0.01, p=.92, \eta^{2}=$ 0.00 , or an Age $\times$ Context interaction, $F(3,131)=0.06, p=.98$, $\eta^{2}=0.01$. Four-year-olds made more within-context errors than 6 -year-olds and young adults, all $p \mathrm{~s}<.005$. No differences were found among the other three age groups, all $p s>.43$.

Similarly, the proportion of foils selected also differed as a function of age, $F(3,131)=15.04, p<.001, \eta^{2}=0.26$, but there was no main effect of context condition, $F(1,131)=2.16, p=$ $.14, \eta^{2}=0.02$, or an Age $\times$ Context interaction, $F(3,131)=0.28$, $p=.84, \eta^{2}=0.01$. Four-year-olds chose foils more frequently than their older counterparts, all $p s<.001$, whereas the other three age groups did not differ, all $p \mathrm{~s}>.99$.

Overselection of foils indicates a potential problem with item memory, thus we tested whether the age differences in relational 
memory accuracy were accounted for by age differences in item memory. A one-way ANCOVA with the proportion of foils selected as a covariate showed that the age effect on relational memory accuracy remained significant in both the similar context condition, $F(3,130)=12.77, p<.001, \eta^{2}=.23$, and the dissimilar context condition, $F(3,130)=12.51, p<.001, \eta^{2}=$ 23 .

Individual differences in aging. Similar to Experiment 1, we tested whether relational memory accuracy was affected by age in our older adult sample, using age as a continuous variable in partial Pearson correlations. In contrast with Experiment 1, the correlation between age and accuracy did not reach significant for either the similar, $r(31)=-.20, p=.26$, or the dissimilar, $r(31)=-.25$, $p=.16$, context condition, after controlling for MMSE and AMNART.

Context-binding errors correlations. Next, we asked whether binding associations to specific contexts in low versus high context similarity relate to each other by correlating acrosscontext errors between the two context conditions. Partial Pearson correlations were conducted for across-context lure selection between the similar and dissimilar context conditions for each age group separately, while controlling for performance on the corresponding verbal IQ task. Interestingly, we found that the acrosscontext errors did not correlate between the two context conditions in 4-year-olds, $r(32)=.28, p=.11$, 6-year-olds, $r(31)=-.15$, $p=.41$, or young adults, $r(31)=-.07, p=.71$. However, the two error rates were positively correlated in older adults, $r(31)=.41$, $p=.02$. The partial correlation held in older adults after further controlling for MMSE scores, $r(30)=.36, p=.047$. These results suggest that binding associations to a specific context with low- or high context similarity may be dissociable from one another in children and young adults but not in older adults (see Figure 7). However, the correlation in older adults only significantly exceeded that in young adults, $z=-1.96, p=.05$, but not in 4-year-olds, $z=-0.58, p=.56$, or 6-year-olds, $z=-1.10, p=$ .27.

Note that our Cronbach's alpha between the two context conditions differed ( 0.77 vs. 0.90$)$, which could potentially exacerbate age-related differences in the correlations between the two across- context error rates. Hence we performed the same analyses using Spearman correlations, which yielded the same results.

\section{Discussion}

In this experiment, we asked how age-related differences on relational memory may vary as a function of context similarity. We found that high context similarity reduced the ability to discern overlapping associations across all age groups. The reduction in accuracy in the similar context condition was accompanied by an increase in association-context binding errors, but not in interitem binding or item memory errors, suggesting our manipulation of context similarity specifically altered relational binding of associations to specific contexts, not overall error rates.

Crucially, different developmental profiles of relational memory transpired depending on the levels of context similarity. For the dissimilar context condition, the age-related differences in relational memory accuracy were strikingly similar to those found in Experiment 1 . There were robust improvements in accuracy between the ages of 4 and 6 , with subtle changes between the age 6 and adulthood, and a decline in old age. In contrast, when relational memory includes the need to discriminate similar contexts, a different age pattern emerged. Six-year-old children no longer outperformed their younger counterparts, and children in both age groups exhibited the lower level of accuracy, concomitant with higher level of across-context errors, compared with young adults. We found almost identical rates of across-context errors between the two age groups of children in the similar context condition, reflecting relational memory deficits throughout early childhood when context inference is high. At the other end of the life spectrum, older adults performed worse than young adults and comparable with 6-year-old children in both context conditions.

Findings from Experiment 2 are consistent with the idea that development of episodic memory does not reach adult-like level until late childhood (reviewed in Ghetti \& Bunge, 2012). Whereas mnemonic discrimination for individual objects appeared to only undergo subtle changes between age 6 and adulthood (Ngo et al., 2018), mnemonic discrimination for more complex representations such as an association within perceptually rich context follows a
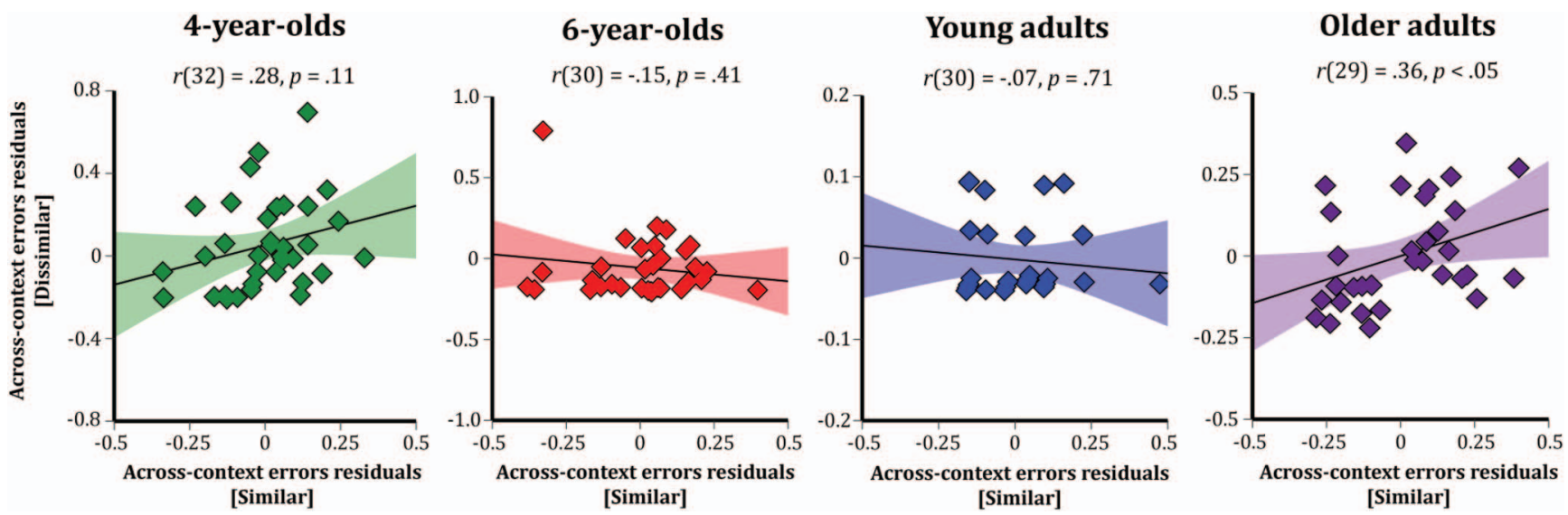

Figure 7. Scatterplots of across-context errors in the similar context condition ( $x$ axes) and the dissimilar context condition ( $y$ axes) in the four age groups. See the online article for the color version of this figure. 
more protracted development, with continuing improvements from age 6 until young adulthood.

Despite the fact that the nature of the test phase was identical, relational binding errors with a low versus high context similarity were also dissociable in children and young adults. However, context-binding errors in the low- and high-context similarity conditions covaried among the older adults. The absence of correlation in three of four groups tested indicates that the aggregation of relational memory and mnemonic discrimination components resulted in dissociable patterns of relational binding errors. The correlation in the older adults in this experiment further corroborates the idea that memory impairments as a result of aging may be overlapping across different facets of episodic memory. However, it is worth noting that unlike Experiment 1, the correlation in older adults only exceeded that of young adults, but not children.

\section{General Discussion}

The current research investigated the development of key components undergirding episodic memory by testing item-level mnemonic discrimination and relational binding as orthogonal tasks (Experiment 1), and relational memory compounded by differential context similarity (Experiment 2 ) in a life span sample. In sum, we found that early childhood is a critical developmental period in which robust gains in item mnemonic discrimination and relational memory occur. Children's abilities to discern overlapping associations learned in similar contexts appears to follow a more protracted development relative to item mnemonic discrimination. All facets of episodic memory examined in this work peak at young adulthood and decline with old age.

\section{Age Differences in Component Processes of Episodic Memory}

An extensive literature has shown that memory improvements in childhood (Lee, Ekstrom, \& Ghetti, 2014; Ofen, 2012; Olson \& Newcombe, 2014) and senescence (Devitt \& Schacter, 2016; Old \& Naveh-Benjamin, 2008) are associated with neurobiological changes both within the hippocampal circuitry and its interconnected memory network. A recent high-resolution MRI study in children aged 6-14 and young adults found increases in volumes of all hippocampal volumes including DG/CA3, CA1-2, and subiculum until early adolescence, with increases in DG/CA3 expanding into young adulthood. Importantly, multivariate analyses that capture the heterogeneous maturation of all hippocampal subfields was found to relate to mnemonic discrimination on the MST (Keresztes et al., 2017). We recently showed that item mnemonic discrimination on the MST in 4- to 8-year-old children was related a gray matter volume in the CA2-4/DG subfields, but not in CA1 or subiculum (Canada, Ngo, Newcombe, Geng, \& Riggins, 2018). Another study showed that DG/CA3 volumes were shown to positively correlate with relational memory performance with children aged 8-14 (Lee et al., 2014).

Evidence from nonhuman primates suggests that hippocampal subfields have different developmental profiles, with the DG showing the most protracted development (Lavenex \& Banta Lavenex, 2013). The protracted development of the DG may give rise to memories with high granularities and increase efficient engagement of hippocampal encoding and retrieval mechanisms
(Lee, Johnson, \& Ghetti, 2017). The maturational course of relational memory dependent on context discrimination may be due to the late developing profiles of the DG, frontal regions, and potentially the long-range white matter connectivity linking the medial temporal lobe to frontal cortex such as the fornix, uncinate fasciculus, and hippocampal cingulum. Thus, behavioral gains in different facets of episodic memory likely arise from not only regional brain changes but also increasingly coordinated activity among these regions (reviewed in Ghetti \& Bunge, 2012).

During aging, the hippocampus undergoes substantial agerelated atrophy (Fraser, Shaw, \& Cherbuin, 2015). Age-related declines in older adults' episodic memory have been linked to structural and functional alterations of the hippocampus (Small, Tsai, Delapaz, Mayeux, \& Stern, 2002). Among older adults, DG/CA3 and subiculum volumes positively correlate with MST lure discrimination (Stark \& Stark, 2017). In addition, worse lure discrimination is associated with an increase in BOLD signal response in the DG/CA3 in older adults (Yassa et al., 2011).

It is important to acknowledge that whereas this work focused on mnemonic discrimination and relational memory, other factors likely contribute to episodic memory development and age-related decline, including strategic control processes at encoding and retrieval, as well as semantic organization (e.g., Ghetti \& Alexander, 2004; reviewed in Bjorklund, Dukes, \& Brown, 2009). Some researchers have sought to explain age differences in episodic memory by appealing to the role of memory strategies in performance, which has been related to maturation/deterioration of prefrontal cortices (Devitt \& Schacter, 2016; Shing et al., 2010). Our task was designed to provide intrinsic support for deep and elaborative encoding by using engaging animations with a strong narrative structure. As such, it is unlikely that differences in strategic control processes alone can explain observed age differences. Future studies should delineate the joint contributions of each component to the wax and wane of episodic memory across the life span.

\section{Relation Between Mnemonic Discrimination and Relational Binding}

It has been long theorized by computational models that the functional role of the hippocampus in episodic memory is multifaceted (Norman \& O'Reilly, 2003). Mnemonic discrimination has been linked to the sparse coding scheme of the granules cells in the dentate gyrus (Neunuebel \& Knierim, 2014), whereas relational binding relies on the conjunctive coding of the recurrent connections among the active units in CA3, which links bind disparate elements from the input layers to a shared representation in CA3 (Nakazawa et al., 2002; Rolls, 2013). Given the uneven maturational profiles across different hippocampal subfields (Keresztes, Ngo, Lindenberger, Werkle-Bergner, \& Newcombe, 2018), we speculate that these regions, along with their interconnected structural and functional brain networks involving regions outside of the hippocampus (Dobbins, Foley, Schacter, \& Wagner, 2002), have distinct developmental trajectories and differentially support distinct aspects of episodic memory. In contrast, the aging literature emphasizes the fact that hippocampal subregions do not function independently, highlighting the circuitry property of the hippocampal formation. Functional decline in any of the hippocampal subregions results in similar memory deficits (Small et 
al., 2002). Further, findings in rodents also showed that lesions to any subregion interrupts global hippocampal function and results in overlapping memory dysfunction (Jarrard, 1993). In agreement with these results, we found that the declines in the two mnemonic components correlate with each other in older adults.

The correlation in older adults also lends support to the idea of dedifferentiation of general intellectual abilities in old age. Correlations between intellectual abilities tend to be higher in aging populations compared with earlier periods of adulthood (Lövdén et al., 2004). A potential mechanism for this increase in the covariance in cognitive processes is the idea of "common cause hypothesis," which posits that age-related decrements in a given cognitive domain (e.g., perceptual speed) may cut across different domains and levels of processing (Baltes \& Lindenberger, 1997). Thus, it is possible that deficits in a given memory function may bleed over to other mnemonic operations. It is also possible that the correlation in mnemonic deficits in older adults reflects individual variations in strategies among older adults. That is, age-related declines in the degree of adaptive strategies employed by older adults resulted in lower capacities in both aspects of episodic memory (Shing et al., 2010).

Our findings on the relation between relational memory and mnemonic discrimination across the life span suggest that the maturation processes of relational memory and item mnemonic discrimination may occur in a relatively dissociable fashion, possibly because of the uneven maturation profiles across different hippocampal subfields (Keresztes et al., 2017, 2018). In contrast, the decline in hippocampally dependent mnemonic processes, including relational memory and mnemonic discrimination, may occur in more unified manner at the tail end of life (Small et al., 2002).

\section{Perceptual Versus Conceptual Similarity}

Item-level mnemonic discrimination in the current research, as is true in most studies employing variants of the MST (reviewed in Yassa et al., 2011), tested fine-grain discrimination for perceptual attributes of objects in the same semantic category. That is, targets and lures differ only in subtle perceptual attributes, such as mugs with slightly different shapes. There are rare circumstances in which perceptual overlap can occur that is divorced from conceptual overlap, that is, objects are perceptually similar but are semantically distant (e.g., pear and light bulb; see Martin, Douglas, Newsome, Man, \& Barense, 2018). The question of whether perceptual or conceptual interference alone, unconfounded by the other dimension, would place a differential demand on hippocampal pattern separation has yet been explored. A fruitful direction for future research is to understand how perceptual versus conceptual inference impact mnemonic discrimination differently, and whether these effects may interact with age across the life span.

\section{Recognition Versus Recall}

The paradigms in the current work employed forced choice recognition tasks, which could potentially limit the implications of our results to other retrieval mechanisms such as yes/no recognition or recall. Age differences have been found to be more robust in a recall task compared with recognition (in development: Levin, Yussen, Pressley, \& de Rose, 1977; in aging: e.g., Craik \& McDowd, 1987). However, recall tasks may not be well suited to test item-level mnemonic discrimination, and could introduce potential confounds such as age differences in language production. Nevertheless, akin to recall, interitem association recognition and mnemonic discrimination of individual items from related lures depend on recollection (reviewed in Yonelinas, 2002). Therefore, although the magnitude in age differences may be attenuated in recognition compared with recall, the general age patterns likely follow a similar trend.

\section{Item Versus Context Mnemonic Discrimination}

Empirical investigations of mnemonic discrimination in humans and animals differ in a fundamental way: in humans, mnemonic discrimination is most often tested using perceptually similar objects or scenes (reviewed in Yassa et al., 2011), whereas studies in animals often emphasize on behaviors dependent on contextual discrimination (reviewed in Hunsaker \& Kesner, 2013). The methodological discrepancy prompts the question of whether separation operates on the item versus context representations in a similar manner. In adult humans, there is evidence to suggest that interference can be minimized by the perirhinal and parahippocampal cortex (in conjunction with the lateral and medial layers of the entorhinal cortex, respectively) as a function of information domain, aiding domain agnostic pattern separation in the DG/CA3. Only the DG/CA3, but not other subfields or other medial temporal lobe cortices, robustly engaged during lure discrimination for both objects and spatial location lures. Furthermore, DG/ CA3 activities correlated with lure discrimination for both objects and spatial location (Reagh \& Yassa, 2014). One study using ultra-high resolution found mnemonic discrimination for similar scene sequences was specifically performed by the DG (Berron et al., 2016). Similarly, other studies that used similar scenes (Bonnici et al., 2012) and complex environments in virtual towns (e.g., Kyle et al., 2015; Stokes et al., 2015) found that the hippocampus carries event- or environment-specific representations. Together, these findings appear to suggest that the hippocampus undertakes domain-general pattern separation.

Here, our findings demonstrate that the development of mnemonic discrimination for item and context may not be homogenous, given that performance on relational memory dependent on successful contextual discrimination appeared to mature later than (a) relational memory in low contextual interference and (b) item-level mnemonic discrimination. It is possible that neural mechanisms required for discerning similar complex representations mature later than those for individual objects. Future studies should investigate mnemonic discrimination mechanisms for content-specific categories (objects and scenes) and conjunctive representations (interitem, item-context bound representations) in development and aging.

\section{Limitations}

Both Experiments 1 and 2 employed a cross-sectional design, which prevents us from understanding the developmental changes of each component process across the life span. Understanding how the changes in both components converge or diverge at different developmental periods would shed light on the relation between mnemonic discrimination and relational binding. In addition, our age range in childhood is relatively restricted by not only practical considerations but also because our focus is to understand the critical age window in which robust gains in episodic memory occur. Nevertheless, earlier (e.g., Newcombe, Balcomb, Ferrara, Hansen, and Koski (2014) and later (e.g., Sastre, Wendelken, Lee, Bunge, \& Ghetti, 2016) develop- 
mental change occurs, and future research should extend the age range to toddlers and to mid- or late childhood.

In Experiment 2, context similarity was manipulated categorically instead of parameterized (in rodents: Leutgeb et al., 2007; in humans: Bonnici et al., 2012; Stokes et al., 2015). It is worth noting that the age patterns for the dissimilar contexts (i.e., indoor vs. outdoors locations) in Experiment 2 are very strikingly similar to the age effects on the relational binding portion of Experiment 1 in which the primary difference between the two contexts are the background colors of the locations (e.g., red and blue houses). It is likely that background color and semantic category serve as equally salient feature of contexts, hence yielding a relatively equivalent level of context interference on relational binding.

Although the paradigms designed by our work appear to be age-appropriate for children and capture sufficient variances in older adults, some portions of the tasks might have been too easy for young adults. In Experiment 1, 6.25\% and 31.25\% among the young adults scored $100 \%$ accuracy in the item mnemonic discrimination and relational binding tasks, respectively. In Experiment $2,18.8 \%$ and $65.6 \%$, of the young adults performed at ceiling in the similar and dissimilar context conditions, respectively. The ceiling effects, particularly in the dissimilar context conditions, could have limited us from detecting age-related differences in relational binding in low context pattern separation demand between 6-year-olds and young adults. Further, the absence of correlation between error types in young adults across two experiments may be attributable to their overall high performance, although it is worth noting that the null correlation in young adults has been previously found in another study with high variance in adults' levels of performance (Ngo et al., 2018).

Future studies should investigate relational memory dependent on context discrimination by parametrically varying the degree of context overlap, potentially on both the perceptual and semantic dimensions, and delineate the developmental trajectories of this process in humans.

\section{Conclusions}

Our study provides important implications on the mechanisms of memory development and senescence across the life span. A comprehensive view of episodic memory - a multifaceted cognitive function - should be studied using a multiprocess approach. This study reveals how the relation between the two components dynamically unfolds over the life span. Although on the surface, the general trends in the rise and fall of episodic memory with age appear to mirror each other, it has been suggested that the decline in aging is not simply development in reverse (Craik \& Bialystok, 2006). A life span description is critical because understanding both the growth and senescence of processes not only mutual benefits both areas of research, but also elucidates the underlying cognitive structure of episodic memory. In a broader context, characterizing the specific type of memory distortions in different developmental populations can inform the educational needs of young children and cater targeted intervention to the elderly. In addition, research in memory maturation and deterioration has implications for how young children and older adults' memory accounts can be evaluated by the legal system.

\section{Context Paragraph}

This set of experiments aimed to decompose episodic memory into its core properties, relational memory and mnemonic discrimination, and to characterize the age-related differences of each component across the human life span. This work was motivated by findings from our previous work (Ngo et al., 2018) showing that performances on these two aspects of episodic memory did not covary in either children or young adulthood. In Experiment 1, instead of using separate tasks, we designed a novel memory task that indexes both processes. Experiment 2 was motivated by the idea that spatial context plays an essential role in episodic memory (for a discussion, see Robin, 2018), and thus we aimed to delineate age-related profiles of mnemonic discrimination for context. The design of this experiment was also influenced by empirical work in the animal literature, taxing the elicitation of different behaviors depending on successful contextual discrimination. Overall, this work shows that relational memory and mnemonic discrimination may be distinct properties of episodic memory, and that the behavioral relationship between the two processes differs at different points in the life span.

\section{References}

Bakker, A., Kirwan, C. B., Miller, M., \& Stark, C. E. (2008). Pattern separation in the human hippocampal CA3 and dentate gyrus. Science, 319, 1640-1642. http://dx.doi.org/10.1126/science.1152882

Baltes, P. B., \& Lindenberger, U. (1997). Emergence of a powerful connection between sensory and cognitive functions across the adult life span: A new window to the study of cognitive aging? Psychology and Aging, 12, 12-21. http://dx.doi.org/10.1037/0882-7974.12.1.12

Berron, D., Schütze, H., Maass, A., Cardenas-Blanco, A., Kuijf, H. J., Kumaran, D., \& Düzel, E. (2016). Strong evidence for pattern separation in human dentate gyrus. The Journal of Neuroscience, 36, 7569-7579. http://dx.doi.org/10.1523/JNEUROSCI.0518-16.2016

Bjorklund, D. F., Dukes, C., \& Brown, R. D. (2009). The development of memory strategies. In M. L. Courage \& N. Cowan (Eds.), Studies in developmental psychology. The development of memory in infancy and childhood (pp. 145-175). New York, NY: Psychology Press.

Bonnici, H. M., Kumaran, D., Chadwick, M. J., Weiskopf, N., Hassabis, D., \& Maguire, E. A. (2012). Decoding representations of scenes in the medial temporal lobes. Hippocampus, 22, 1143-1153. http://dx.doi.org/ 10.1002/hipo. 20960

Canada, K. L., Ngo, C. T., Newcombe, N. S., Geng, F., \& Riggins, T. (2018). It's all in the details: Relations between young children's developing pattern separation abilities and hippocampal subfield volumes. Cerebral Cortex. Advance online publication. http://dx.doi.org/10.1093/ cercor/bhy 211

Castel, A. D., \& Craik, F. I. (2003). The effects of aging and divided attention on memory for item and associative information. Psychology and Aging, 18, 873-885. http://dx.doi.org/10.1037/0882-7974.18.4.873

Chadwick, M. J., Bonnici, H. M., \& Maguire, E. A. (2014). CA3 size predicts the precision of memory recall. Proceedings of the National Academy of Sciences of the United States of America, 111, 1072010725. http://dx.doi.org/10.1073/pnas.1319641111

Cowan, N., Naveh-Benjamin, M., Kilb, A., \& Saults, J. S. (2006). Lifespan development of visual working memory: When is feature binding difficult? Developmental Psychology, 42, 1089-1102. http://dx.doi.org/ 10.1037/0012-1649.42.6.1089

Craik, F. I. M., \& Bialystok, E. (2006). Cognition through the lifespan: Mechanisms of change. Trends in Cognitive Sciences, 10, 131-138. http://dx.doi.org/10.1016/j.tics.2006.01.007 
Craik, F. I. M., \& McDowd, J. M. (1987). Age differences in recall and cognition. Journal of Experimental Psychology: Learning, Memory, and Cognition, 13, 474-479. http://dx.doi.org/10.1037/0278-7393.13.3.474

Davachi, L. (2006). Item, context and relational episodic encoding in humans. Current Opinion in Neurobiology, 16, 693-700. http://dx.doi .org/10.1016/j.conb.2006.10.012

Dennis, N. A., Bowman, C. R., \& Peterson, K. M. (2014). Age-related differences in the neural correlates mediating false recollection. Neurobiology of Aging, 35, 395-407. http://dx.doi.org/10.1016/j.neurobiolaging.2013.08.019

Devitt, A. L., \& Schacter, D. L. (2016). False memories with age: Neural and cognitive underpinnings. Neuropsychologia, 91, 346-359. http://dx .doi.org/10.1016/j.neuropsychologia.2016.08.030

Dobbins, I. G., Foley, H., Schacter, D. L., \& Wagner, A. D. (2002). Executive control during episodic retrieval: Multiple prefrontal processes subserve source memory. Neuron, 35, 989-996. http://dx.doi.org/ 10.1016/S0896-6273(02)00858-9

Fandakova, Y., Shing, Y. L., \& Lindenberger, U. (2013). Differences in binding and monitoring mechanisms contribute to lifespan age differences in false memory. Developmental Psychology, 49, 1822-1832. http://dx.doi.org/10.1037/a0031361

Folstein, M. F., Folstein, S. E., \& McHugh, P. R. (1975). "Mini-mental state": A practical method for grading the cognitive state of patients for the clinician. Journal of Psychiatric Research, 12, 189-198. http://dx .doi.org/10.1016/0022-3956(75)90026-6

França, T. F. A., Bitencourt, A. M., Maximilla, N. R., Barros, D. M., \& Monserrat, J. M. (2017). Hippocampal neurogenesis and pattern separation: A meta-analysis of behavioral data. Hippocampus, 27, 937-950. http://dx.doi.org/10.1002/hipo.22746

Fraser, M. A., Shaw, M. E., \& Cherbuin, N. (2015). A systematic review and meta-analysis of longitudinal hippocampal atrophy in healthy human ageing. NeuroImage, 112, 364-374. http://dx.doi.org/10.1016/j .neuroimage.2015.03.035

Ghetti, S., \& Alexander, K. W. (2004). "If it happened, I would remember it": Strategic use of event memorability in the rejection of false autobiographical events. Child Development, 75, 542-561. http://dx.doi.org/ 10.1111/j.1467-8624.2004.00692.x

Ghetti, S., \& Bunge, S. A. (2012). Neural changes underlying the development of episodic memory during middle childhood. Developmental Cognitive Neuroscience, 2, 381-395. http://dx.doi.org/10.1016/j.den .2012 .05 .002

Grober, E., \& Sliwinsk, M. (1991). Development and validation of a model for estimating premorbid verbal intelligence in the elderly. Journal of Clinical and Experimental Neuropsychology, 13, 933-949. http://dx.doi .org/10.1080/01688639108405109

Holden, H. M., Toner, C., Pirogovsky, E., Kirwan, C. B., \& Gilbert, P. E. (2013). Visual object pattern separation varies in older adults. Learning \& Memory, 20, 358-362. http://dx.doi.org/10.1101/lm.030171.112

Horner, A. J., \& Doeller, C. F. (2017). Plasticity of hippocampal memories in humans. Current Opinion in Neurobiology, 43, 102-109. http://dx.doi .org/10.1016/j.conb.2017.02.004

Hunsaker, M. R., \& Kesner, R. P. (2013). The operation of pattern separation and pattern completion processes associated with different attributes or domains of memory. Neuroscience and Biobehavioral Reviews, 37, 36-58. http://dx.doi.org/10.1016/j.neubiorev.2012.09.014

Jarrard, L. E. (1993). On the role of the hippocampus in learning and memory in the rat. Behavioral \& Neural Biology, 60, 9-26. http://dx .doi.org/10.1016/0163-1047(93)90664-4

Kaufman, A. S., \& Kaufman, N. L. (1990). Kaufman Brief Intelligence Test. Circle Pines, MN: American Guidance Service.

Keresztes, A., Bender, A. R., Bodammer, N. C., Lindenberger, U., Shing, Y. L., \& Werkle-Bergner, M. (2017). Hippocampal maturity promotes memory distinctiveness in childhood and adolescence. Proceedings of the National Academy of Sciences of the United States of America, 114, 9212-9217. http://dx.doi.org/10.1073/pnas.1710654114

Keresztes, A., Ngo, C. T., Lindenberger, U., Werkle-Bergner, M., \& Newcombe, N. S. (2018). Hippocampal maturation drives memory from generalization to specificity. Trends in Cognitive Sciences, 22, 676-686. http://dx.doi.org/10.1016/j.tics.2018.05.004

Kirwan, C. B., \& Stark, C. E. L. (2007). Overcoming interference: An fMRI investigation of pattern separation in the medial temporal lobe. Learning \& Memory, 14, 625-633. http://dx.doi.org/10.1101/lm.663507

Kyle, C. T., Stokes, J. D., Lieberman, J. S., Hassan, A. S., \& Ekstrom, A. D. (2015). Successful retrieval of competing spatial environments in humans involves hippocampal pattern separation mechanisms. eLife, 4, e10499. http://dx.doi.org/10.7554/eLife.10499

Lacy, J. W., Yassa, M. A., Stark, S. M., Muftuler, L. T., \& Stark, C. E. (2011). Distinct pattern separation related transfer functions in human CA3/dentate and CA1 revealed using high-resolution fMRI and variable mnemonic similarity. Learning \& Memory, 18, 15-18. http://dx.doi.org/ 10.1101/lm.1971111

Lavenex, P., \& Banta Lavenex, P. (2013). Building hippocampal circuits to learn and remember: Insights into the development of human memory. Behavioural Brain Research, 254, 8-21. http://dx.doi.org/10.1016/j.bbr .2013.02.007

Lebel, C., Gee, M., Camicioli, R., Wieler, M., Martin, W., \& Beaulieu, C. (2012). Diffusion tensor imaging of white matter tract evolution over the lifespan. NeuroImage, 60, 340-352. http://dx.doi.org/10.1016/j neuroimage.2011.11.094

Lee, J. K., Ekstrom, A. D., \& Ghetti, S. (2014). Volume of hippocampal subfields and episodic memory in childhood and adolescence. NeuroImage, 94, 162-171. http://dx.doi.org/10.1016/j.neuroimage.2014.03.019

Lee, J. K., Johnson, E. G., \& Ghetti, S. (2017). Hippocampal development: Structure, function and implications. In D. Hannula \& M. Duff (Eds.), The hippocampus from cells to systems (pp. 141-166). Cham, UK: Springer. http://dx.doi.org/10.1007/978-3-319-50406-3_6

Leutgeb, J. K., Leutgeb, S., Moser, M. B., \& Moser, E. I. (2007). Pattern separation in the dentate gyrus and CA3 of the hippocampus. Science, 315, 961-966. http://dx.doi.org/10.1126/science.1135801

Levin, J. R., Yussen, S. R., Pressley, M., \& de Rose, T. M. (1977) Developmental changes in assessing recall and recognition memory capacity. Developmental Psychology, 13, 608-615. http://dx.doi.org/10 $.1037 / 0012-1649.13 .6 .608$

Lloyd, M. E., Doydum, A. O., \& Newcombe, N. S. (2009). Memory binding in early childhood: Evidence for a retrieval deficit. Child Development, 80, 1321-1328. http://dx.doi.org/10.1111/j.1467-8624.2009 .01353.x

Lövdén, M., Ghisletta, P., \& Lindenberger, U. (2004). Cognition in the Berlin Aging Study (BASE): The first 10 years. Aging, Neuropsychology, and Cognition, 11, 104-133. http://dx.doi.org/10.1080/ 13825580490510982

Martin, C. B., Douglas, D., Newsome, R. N., Man, L. L., \& Barense, M. D. (2018). Integrative and distinctive coding of visual and conceptual object features in the ventral visual stream. eLife, 7, e31873. http://dx.doi.org/ 10.7554/eLife. 31873

McClelland, J. L., McNaughton, B. L., \& O’Reilly, R. C. (1995). Why there are complementary learning systems in the hippocampus and neocortex: Insights from the successes and failures of connectionist models of learning and memory. Psychological Review, 102, 419-457. http://dx.doi.org/10.1037/0033-295X.102.3.419

McHugh, T. J., Jones, M. W., Quinn, J. J., Balthasar, N., Coppari, R., Elmquist, J. K., . . . Tonegawa, S. (2007). Dentate gyrus NMDA receptors mediate rapid pattern separation in the hippocampal network. Science, 317, 94-99. http://dx.doi.org/10.1126/science.1140263

Nakazawa, K., Quirk, M. C., Chitwood, R. A. R. A., Watanabe, M., Yeckel, M. F. M. F., Sun, L. D. L. D., . . Tonegawa, S. (2002). Requirement for 
hippocampal CA3 NMDA receptors in associative memory recall. Science, 297, 211-218. http://dx.doi.org/10.1126/science.1071795

Naveh-Benjamin, M. (2000). Adult age differences in memory performance: Tests of an associative deficit hypothesis. Journal of Experimental Psychology: Learning, Memory, and Cognition, 26, 1170-1187. http://dx.doi.org/10.1037/0278-7393.26.5.1170

Nelson, C. A. (1995). The ontogeny of human memory: A cognitive neuroscience perspective. Developmental Psychology, 31, 723-738. http://dx.doi.org/10.1037/0012-1649.31.5.723

Neunuebel, J. P., \& Knierim, J. J. (2014). CA3 retrieves coherent representations from degraded input: Direct evidence for CA3 pattern completion and dentate gyrus pattern separation. Neuron, 81, 416-427. http://dx.doi.org/10.1016/j.neuron.2013.11.017

Newcombe, N. S., Balcomb, F., Ferrara, K., Hansen, M., \& Koski, J. (2014). Two rooms, two representations? Episodic-like memory in toddlers and preschoolers. Developmental Science, 17, 743-756. http://dx .doi.org/10.1111/desc. 12162

Ngo, C. T., Newcombe, N. S., \& Olson, I. R. (2018). The ontogeny of relational memory and pattern separation. Developmental Science, 21(2), e12556. http://dx.doi.org/10.1111/desc. 12556

Norman, K. A., \& O'Reilly, R. C. (2003). Modeling hippocampal and neocortical contributions to recognition memory: A complementarylearning-systems approach. Psychological Review, 110, 611-646. http:// dx.doi.org/10.1037/0033-295X.110.4.611

Ofen, N. (2012). The development of neural correlates for memory formation. Neuroscience and Biobehavioral Reviews, 36, 1708-1717. http://dx.doi.org/10.1016/j.neubiorev.2012.02.016

Old, S. R., \& Naveh-Benjamin, M. (2008). Differential effects of age on item and associative measures of memory: A meta-analysis. Psychology and Aging, 23, 104-118. http://dx.doi.org/10.1037/0882-7974.23.1.104

Olson, I. R., \& Newcombe, N. S. (2014). Binding together the elements of episodes: Relational memory and the developmental trajectory of the hippocampus. In P. J. Bauer and R. Fivush (Eds.), The Wiley handbook and the development of children's memory (Vol. I, pp. 285-308). New York, NY: Wiley.

O'Reilly, R. C., \& McClelland, J. L. (1994). Hippocampal conjunctive encoding, storage, and recall: Avoiding a trade-off. Hippocampus, 4, 661-682. http://dx.doi.org/10.1002/hipo.450040605

Reagh, Z. M., \& Yassa, M. A. (2014). Object and spatial mnemonic interference differentially engage lateral and medial entorhinal cortex in humans. Proceedings of the National Academy of Sciences of the United States of America, 111, E4264-E4273. http://dx.doi.org/10.1073/pnas .1411250111

Riggins, T. (2014). Longitudinal investigation of source memory reveals different developmental trajectories for item memory and binding. Developmental Psychology, 50, 449-459. http://dx.doi.org/10.1037/a0033622

Robin, J. (2018). Spatial scaffold effects in event memory and imagination. WIREs Cognitive Science, 9, e1462. http://dx.doi.org/10.1002/wcs.1462

Rollins, L., \& Cloude, E. B. (2018). Development of mnemonic discrimination during childhood. Learning \& Memory, 25, 294-297. http://dx .doi.org/10.1101/lm.047142.117

Rolls, E. T. (1996). A theory of hippocampal function in memory. Hippocampus, 6, 601-620. http://dx.doi.org/10.1002/(SICI)10981063(1996)6:6<601::AID-HIPO5>3.0.CO;2-J

Rolls, E. T. (2013). The mechanisms for pattern completion and pattern separation in the hippocampus. Frontiers in Systems Neuroscience, 7 , 74. http://dx.doi.org/10.3389/fnsys.2013.00074

Rugg, M. D., \& King, D. R. (2018). Ventral lateral parietal cortex and episodic memory retrieval. Cortex, 107, 238-250.

Sastre, M., III, Wendelken, C., Lee, J. K., Bunge, S. A., \& Ghetti, S. (2016). Age- and performance-related differences in hippocampal con- tributions to episodic retrieval. Developmental Cognitive Neuroscience, 19, 42-50. http://dx.doi.org/10.1016/j.den.2016.01.003

Serres, L. (2001). Morphological changes of the human hippocampal formation from midgestation to early childhood. In C. A. Nelson \& M. Luciania (Eds.), Handbook of developmental cognitive neuroscience (pp. 45-58). Cambridge, MA: MIT Press.

Shing, Y. L., Werkle-Bergner, M., Brehmer, Y., Müller, V., Li, S. C., \& Lindenberger, U. (2010). Episodic memory across the lifespan: The contributions of associative and strategic components. Neuroscience and Biobehavioral Reviews, 34, 1080-1091. http://dx.doi.org/10.1016/j .neubiorev.2009.11.002

Sluzenski, J., Newcombe, N. S., \& Kovacs, S. L. (2006). Binding, relational memory, and recall of naturalistic events: A developmental perspective. Journal of Experimental Psychology: Learning, Memory, and Cognition, 32, 89-100. http://dx.doi.org/10.1037/0278-7393.32.1.89

Small, S. A., Tsai, W. Y., DeLaPaz, R., Mayeux, R., \& Stern, Y. (2002). Imaging hippocampal function across the human life span: Is memory decline normal or not? Annals of Neurology, 51, 290-295. http://dx.doi .org/10.1002/ana.10105

Stark, S. M., \& Stark, C. E. L. (2017). Age-related deficits in the mnemonic similarity task for objects and scenes. Behavioural Brain Research, 333, 109-117. http://dx.doi.org/10.1016/j.bbr.2017.06.049

Stark, S. M., Yassa, M. A., Lacy, J. W., \& Stark, C. E. (2013). A task to assess behavioral pattern separation (BPS) in humans: Data from healthy aging and mild cognitive impairment. Neuropsychologia, 51, 24422449. http://dx.doi.org/10.1016/j.neuropsychologia.2012.12.014

Stokes, J., Kyle, C., \& Ekstrom, A. D. (2015). Complementary roles of human hippocampal subfields in differentiation and integration of spatial context. Journal of Cognitive Neuroscience, 27, 546-559. http://dx.doi .org/10.1162/jocn_a_00736

Tombaugh, T. N., \& McIntyre, N. J. (1992). The mini-mental state examination: A comprehensive review. Journal of the American Geriatrics Society, 40, 922-935. http://dx.doi.org/10.1111/j.1532-5415.1992 .tb01992.x

Tulving, E. (2002). Episodic memory: From mind to brain. Annual Review of Psychology, 53, 1-25. http://dx.doi.org/10.1146/annurev.psych.53 100901.135114

Wheeler, M. A., Stuss, D. T., \& Tulving, E. (1997). Toward a theory of episodic memory: The frontal lobes and autonoetic consciousness. Psychological Bulletin, 121, 331-354. http://dx.doi.org/10.1037/0033-2909 121.3.331

Yassa, M. A., Lacy, J. W., Stark, S. M., Albert, M. S., Gallagher, M., \& Stark, C. E. (2011). Pattern separation deficits associated with increased hippocampal CA3 and dentate gyrus activity in nondemented older adults. Hippocampus, 21, 968-979.

Yeung, L. K., Ryan, J. D., Cowell, R. A., \& Barense, M. D. (2013). Recognition memory impairments caused by false recognition of novel objects. Journal of Experimental Psychology: General, 142, 1384-1397. http://dx.doi.org/10.1037/a0034021

Yim, H., Dennis, S. J., \& Sloutsky, V. M. (2013). The development of episodic memory: Items, contexts, and relations. Psychological Science, 24, 2163-2172. http://dx.doi.org/10.1177/0956797613487385

Yonelinas, A. P. (2002). The nature of recollection and familiarity: A review of 30 years of research. Journal of Memory and Language, 46, 441-517.

Received April 2, 2018

Revision received January 4, 2019 Accepted January 14, 2019 